INRA Prod. Anim., 2005, 18 (5), 323-338

\section{Pour un élevage ovin allaitant performant et durable sur parcours : un système expérimental sur le Causse du Larzac}

\author{
G. MOLENAT', D. FOULQUIE', P. AUTRAN², J. BOUIX'3, D. HUBERT ${ }^{1}$, M. JACQUIN ${ }^{3}$ \\ F. BOCQUIER ${ }^{1}, B . B I B E^{3}$ \\ 1 INRA, Elevage des Ruminants en Régions Chaudes, 2 place Viala, F- 34060 Montpellier cedex 01 \\ 2 INRA, Domaine de La Fage, F-12250 Roquefort \\ 3 INRA, Station d'Amélioration Génétique des Animaux, F-31326 Castanet-Tolosan cedex \\ courriel :bouix@toulouse.inra.fr
}

\begin{abstract}
Sur les parcours du Causse du Larzac, une recherche pluridisciplinaire aboutit à la mise en place d'un système de production ovin allaitant en plein air intégral avec un haut niveau de performances. Ce système, basé sur l'utilisation prioritaire et l'entretien des parcours, innove grâce à un allongement de la période de pâturage printanière obtenu par la fertilisation modérée d'une très faible proportion de la surface, et à l'introduction de la brebis INRA 401 à la fois productive et bien adaptée à un environnement contraignant.
\end{abstract}

Les végétations steppiques du Causse du Larzac sont des ressources pastorales tardives au printemps, séchantes et disponibles seulement sur une brève période. Longtemps ressources saisonnières, elles n’ont plus représenté, après l'exode rural du début du $20^{\mathrm{e}}$ siècle, qu'un appoint occasionnel pour l'élevage ovin local. Leur utilité devenait accessoire dans un contexte de conduites de troupeaux de plus en plus intensives.

Dès la décennie 1970-80, à l'instar des autres territoires pastoraux secs, la marginalité de ces espaces est apparue comme un non sens écologique, économique et social, ce qui justifiait des recherches pour reprendre leur valorisation et leur entretien au moyen principalement de l'élevage ovin.

Ainsi, en 1972, l'INRA a entrepris un programme expérimental visant à préciser les conditions de l'établissement d'un élevage ovin allaitant économe sur les parcours du Causse du Larzac, au domaine de La Fage (Molénat et al 1976, Groupe de recherches INRACNRS 1979). D'emblée, l'objectif primordial a été de privilégier l'usage des ressources spontanées. Il s'agissait d'élaborer un système technique de production cohérent dont la pérennité reposerait sur la reproductibilité d'une utilisation suffisamment intense des ressources pastorales. L'enjeu était de parvenir à des formes durables de gestion des végétations qui allient les exigences de la production à celles de l'entretien de l'environnement. Un bon niveau de production animale, associé à la réduction des charges et des intrants, devait en outre garantir les marges économiques indispensables.

C'est donc un élevage à finalités multiples qu'il fallait concevoir, avec des animaux adaptés aux conditions difficiles et des modes de conduite appropriés. En conséquence, le programme pluridisciplinaire s'est structuré autour de trois thématiques. La première concerne l'amélioration des connaissances sur les potentialités des parcours afin d'en définir les conditions d'exploitation et la seconde la recherche d'un type d'animal capable d'une forte productivité individuelle augurant des marges élevées par brebis. Les deux convergent vers une synthèse consistant à élaborer, à l'aide d'un troupeau expérimental, une conduite d'élevage répondant aux critères retenus.

Cet article exposera d'abord les principales conclusions obtenues à propos de ces trois thématiques afin de montrer comment se sont articulées les étapes de la construction du système. Ensuite, la gestion des végétations pâturées au printemps fera l'objet de développements particuliers car elle s'est révélée être une des clés du cycle zootechnique. Enfin, la cohérence d'ensemble sera examinée afin de resituer les résultats par rapport au contexte et aux questionnements plus globaux concernant les milieux pastoraux et aussi afin de dégager la part d'innovation que recèle ce travail. Dans ces milieux, les considérations liées à l'entretien du milieu ou à la notion d'autonomie fourragère (Louault et al 1998) offrent par exemple un cadre original d'interprétation des conduites alimentaires, cadre devenu aujourd'hui incontournable. En outre, l'ambition est aussi de montrer comment la pluridisciplinarité sur le long terme, a rendu possible l'élaboration de réponses aux défis générés par les multiples interactions entre les nombreux facteurs pris en considération et de tenir compte d'éléments connexes de nature économique et sociale (investissements, travail etc.). Il s'agit encore de montrer comment, en conséquence, un tel programme contribue à éclairer les options stratégiques en matière d'élevage, de sélection animale, de choix des races et d'aménagement dans les milieux pastoraux. 


\section{1 / Elaboration d'un sys- tème de production ovin allaitant performant sur parcours}

\section{1 / Donner la priorité à l'usage des ressources pastorales}

Sur les parcours du Causse du Larzac (altitude moyenne $800 \mathrm{~m}$ ), la pelouse clairsemée recouvre 75 à $80 \%$ de la surface, le reste étant constitué de couverts ligneux dominés par le buis. La végétation herbacée atteint son maximum de production au printemps. Tardive, elle démarre début mai, puis se développe rapidement ; elle termine son cycle entre la mi-juin et juillet puis subit la sécheresse estivale sans dommages pour sa pérennité. A la faveur des pluies de fin d'été et d'automne se produit généralement une repousse modeste.

Le printemps offre la perspective la plus sûre d'une herbe abondante de qualité. Pour réaliser les expérimentations, l'agnelage a donc été placé au printemps de manière à privilégier l'usage des végétations spontanées pendant l'allaitement, époque la plus exigeante du cycle d'élevage. La brièveté de la période favorable, rarement plus de 30 jours, aurait imposé toutefois des aliments ou des compléments exogènes pour garantir de bonnes croissances d'agneaux et aurait donc réduit considérablement l'intérêt des parcours. Cette difficulté a pu être aplanie grâce à la perspective d'allongement de la période d'alimentation des brebis allaitantes sur parcours, ouverte par les résultats des études sur la dynamique de réponse de la végétation native à un accroissement de la fertilité des sols (Langlet et Hubert 1985).

A partir de la décennie 1970, une étude approfondie des végétations de Causse, en conditions pâturées, a ainsi été menée sur les parcours du domaine de La Fage, en même temps qu'étaient expérimentées des procédures d'élevage en plein air et qu'étaient réalisées les études de génétique animale. Les 280 ha de parcours du domaine ont été divisés en parcelles clôturées de manière à atteindre une bonne maîtrise des conditions de pâturage. Après des essais préliminaires de fertilisation, l'une des parcelles a été fertilisée de façon régulière ; divisée en parcelles plus petites et pâturée en rotation, elle a fait l'objet d'observations à caractère agronomique (et aussi à caractère éco- logique). Il en est résulté la pratique en routine d'une fertilisation des végétations spontanées dans le but de sécuriser l'alimentation des brebis allaitantes. La connaissance des végétations natives et des végétations fertilisées a ouvert la voie à un travail sur le calendrier d'alimentation des brebis au printemps et sur les logiques d'utilisation des ressources.

\section{a) La végétation pastorale native}

La végétation native $(\mathbf{N})$ de la pelouse, relevée le long de transects permanents de 100 points, est constituée de rares légumineuses (5 à $15 \%$ ) et surtout de graminées dont les principales sont : Bromus erectus (30 à $50 \%$ ), Brachypodium pinnatum (8 à $15 \%$ ), Festuca duriuscula (5 à $15 \%$ ), Stipa pennata (7 à $20 \%$ ) et Festuca rubra (5 à $15 \%$ ) (relevés des printemps 20002003).

La production printanière, mesurée sur une série de 14 années a varié entre

Tableau 1. Production de la biomasse herbacée des parcours fertilisés et natifs selon les années (mesurée à la fin de mai, de juillet et d'octobre).

\begin{tabular}{|c|c|c|c|c|c|c|}
\hline & \multicolumn{6}{|c|}{ Quantité de biomasse herbacée produite (kg MS / ha) } \\
\hline & \multicolumn{2}{|c|}{ avril - mai } & \multicolumn{2}{|c|}{ juin - juillet } & \multicolumn{2}{|c|}{ septembre - octobre } \\
\hline & natif & fertilisé & natif & fertilisé & natif & fertilisé \\
\hline 1980 & - & 1180 & - & 1600 & - & 800 \\
\hline 1981 & - & 2127 & - & 1565 & - & 723 \\
\hline 1982 & - & 1475 & - & 733 & - & 1480 \\
\hline 1983 & - & 2474 & - & 1270 & - & 1526 \\
\hline 1984 & - & 1609 & - & 1234 & - & 961 \\
\hline 1985 & - & 2579 & - & 926 & - & 0 \\
\hline 1986 & - & 1138 & - & 225 & - & 516 \\
\hline 1987 & - & 2335 & - & 1394 & - & 1170 \\
\hline 1988 & - & 3132 & - & 810 & - & 680 \\
\hline 1989 & - & 2509 & - & 396 & - & 644 \\
\hline Moyenne & & 2056 & & 1015 & & 850 \\
\hline Ecart-type & & 670 & & 476 & & 459 \\
\hline 1990 & 582 & 3738 & 400 & 922 & 96 & 696 \\
\hline 1991 & 592 & 2467 & 424 & 826 & 178 & 1022 \\
\hline 1992 & 949 & 3911 & 439 & 1156 & 137 & 712 \\
\hline 1993 & 1117 & 4771 & 709 & 1551 & 156 & 621 \\
\hline 1994 & 1307 & 5310 & 343 & 487 & 193 & 950 \\
\hline 1995 & 1148 & 5433 & 246 & 389 & 190 & 1207 \\
\hline 1996 & 892 & 4952 & 357 & 571 & 164 & 915 \\
\hline 1997 & 763 & 3257 & 703 & 1200 & 275 & 1247 \\
\hline 1998 & 903 & 4032 & 508 & 1009 & 196 & 994 \\
\hline 1999 & 1168 & 5022 & 184 & 199 & 187 & 1178 \\
\hline 2000 & 1345 & 4153 & 479 & 869 & 203 & 1026 \\
\hline 2001 & 1161 & 6253 & 760 & 908 & 160 & 981 \\
\hline 2002 & 1068 & 4075 & 623 & 692 & 350 & 1606 \\
\hline 2003 & 984 & 4863 & 0 & 0 & 259 & 542 \\
\hline $\begin{array}{c}\text { Moyenne } \\
1990-2003\end{array}$ & 999 & 4446 & 441 & 770 & 196 & 978 \\
\hline Ecart-type & 237 & 975 & 215 & 416 & 63 & 282 \\
\hline
\end{tabular}

582 et $1345 \mathrm{~kg}$ MS/ha selon les années, se situant le plus fréquemment aux environs de $1000 \mathrm{~kg} \mathrm{MS} / \mathrm{ha}$ (tableau 1). Le démarrage ne se produit guère avant le 10 mai, l'époque de pleine production se situant à la fin mai. Dès juillet, les graminées épient, et en août l'herbe est de plus en plus sèche. A l'automne, la repousse est très variable en fonction de l'année : de 100 à $350 \mathrm{~kg} \mathrm{MS} / \mathrm{ha}$ autour d'une moyenne n'excédant pas 200 kg (Hubert et Grandjanny 1989).

La valeur nutritive de l'herbe disponible, estimée par des analyses fourragères, montre vers la fin mai des teneurs en Matières Azotées Totales (MAT) de $125 \mathrm{~g} / \mathrm{kg}$ MS et des valeurs énergétiques calculées de $0,80 \mathrm{UFL} / \mathrm{kg}$ MS. Ces teneurs se maintiennent jusque vers le 15 juin, puis décroissent ensuite régulièrement pour atteindre 85 $\mathrm{g} \mathrm{MAT} / \mathrm{kg}$ MS et $0,73 \mathrm{UFL} / \mathrm{kg}$ MS à la fin juillet (observations effectuées entre 1995 et 1999 : Hubert non publié). 


\section{b) La végétation pastorale fertilisée}

Sur le Causse, les terrains de parcours se présentent comme une mosaïque de sols aux potentialités diverses. Les zones les plus favorables, souvent cultivées dans le passé, puis abandonnées parce que mal situées et trop exiguës, ont finalement été colonisées par la végétation spontanée avoisinante. Elles se sont montrées capables, dans des essais en petites parcelles, de porter une végétation herbacée plus précoce et plus productive sous l'effet d'une fertilisation modérée (Groupe de recherches INRA-CNRS 1979, Langlet 1979).

Pour une fertilisation en routine dans une grande parcelle, les doses annuelles retenues ont ensuite été de $40 \mathrm{~kg}$ d'acide phosphorique et $65 \mathrm{~kg}$ d'azote/ha. En conditions pâturées, les évolutions constatées sur le long terme ont révélé l'aptitude au renouvellement soutenu de la production herbacée. Après une augmentation progressive durant les premières années de fertilisation, la production mesurée à la fin mai a oscillé, en fonction des variations climatiques inter-annuelles, autour de $4000 \mathrm{~kg} \mathrm{MS} / \mathrm{ha}$ (tableau 1).

La végétation fertilisée $(\mathbf{F})$, principalement des graminées, est constituée de Bromus erectus (30 à $50 \%$ ), Poa pratensis (5 à $10 \%)$,Vulpia myuros (15 à $40 \%$ ), et Bromus mollis (10 à $20 \%$ ), auxquelles il faut ajouter jusqu'à $12 \%$ d'herbe sèche de l'automne précédent (relevés des printemps 2000-2003). Sous l'effet conjugué de la fertilisation et du pâturage, des espèces comme Brachypodium pinnatum et Festuca duriuscula ont fortement régressé au profit de Poa pratensis d'une part et de Vulpia myuros d'autre part. Les mesures effectuées chaque année au printemps n'ont pas révélé de modification du nombre d'espèces (50 espèces sous 800 points) et l'indice d'équitabilité s'est maintenu $(1,08 \pm 0,06$ en 1978 et $1,03 \pm 0,09$ en $2003 \div$ : Hubert et Alard publication en préparation).

Vers la mi-avril, les graminées annuelles fertilisées ont un développement suffisant pour être pâturées : Vulpia myuros est la plus précoce, suivie par Bromus mollis puis par Bromus erectus (graminée pérenne) qui atteint son pic de production lorsque Vulpia myuros commence à épier.

L'échelonnement de production des espèces détermine ainsi une végétation active et feuillue entre mi-avril et mimai, une précocité provocant une extension de l'offre pastorale vers le début du printemps. Cependant la qualité de cette ressource décroît après la fin mai aussi rapidement que celle des végétations natives : $200 \mathrm{~g}$ MAT et $0,98 \mathrm{UFL} / \mathrm{kg}$ MS au 20 avril puis $80 \mathrm{~g}$ MAT et $0,69 \mathrm{UFL} / \mathrm{kg}$ MS à la fin juillet (observations effectuées entre 1995 et 1999 : Hubert non publié).

A l'automne, la repousse, également très variable, produit sensiblement $1000 \mathrm{~kg}$ MS/ ha en moyenne, sauf en année très sèche.

c) Combiner pelouse native et pelouse fertilisée pour asseoir une conduite alimentaire

Sur la végétation fertilisée, une mise à l'herbe en avance de 3 semaines environ au printemps sur celle de la végétation native est envisageable. L'allongement de la période, où l'abondance et la qualité de l'herbe se conjuguent, est de nature à faire sauter le verrou du manque d'herbe au début du printemps. Combiner des parcours fertilisés et des parcours natifs, c'est en effet l'assurance de 2 mois d'herbe de qualité entre mi-avril et mi-juin. Avec un agnelage au début d'avril pour un sevrage en début d'été lorsque la végétation commence à se dessécher, les agneaux peuvent être allaités par des mères alimentées exclusivement sur parcours.

Un élevage ovin allaitant basé prioritairement sur des ressources pastorales caussenardes devient dès lors concevable avec la perspective de 50 à 60 jours d'herbe en croissance au printemps (allaitement), puis à nouveau des repousses pendant 40 à 50 jours à l'automne (lutte), et des végétations mâtures plus ou moins sèches pendant une bonne partie du reste de l'année.

\section{2 / Les animaux et la conduite du troupeau}

Très tôt dans le programme, le profil de l'animal recherché a inclus la productivité comme un moyen de réduire la vulnérabilité du système de production face aux fluctuations du contexte général de l'élevage ovin. Ce choix de la productivité trouve aussi son inspiration dans la taille limitée des troupeaux et des exploitations, y compris sur parcours, en raison de la faible disponibilité de surfaces dans nos pays. Un animal productif offre des perspectives de compétitivité face aux aléas des marchés et à ceux des systèmes d'aides extérieures et de subventions. Les indications qu'il fournit sur l'aptitude des pâturages à répondre à de fortes exigen- ces zootechniques peuvent en outre être d'un grand intérêt. En évaluant des situations typées peu communes, le programme de recherche se place en situation de produire des informations utilisables dans des modèles analytiques ou prédictifs. Concrètement, c'est la possibilité de produire un plus grand nombre d'agneaux par mère qui a été retenue, sachant que c'est au sevrage que cette production est évaluée. Il s'agit en effet de faire en sorte que l'herbe des parcours ait une contribution maximale à la phase principale d'élaboration du produit final qu'est l'agneau de boucherie.

La pérennité du système de production dépend aussi des méthodes d'élevage qui ont été voulues économes en intégrant des facteurs sociaux comme le travail par exemple (Rouquette et al 1997) et en optant pour le plein air permanent afin de réduire les charges de structure. Les pratiques et procédures ont été élaborées avec le souci de la simplicité, de l'efficacité et de manière à limiter les interventions humaines en laissant une grande autonomie à l'animal. La priorité donnée à l'exploitation des ressources pastorales a influencé le calendrier d'alimentation mais aussi un grand nombre de procédures de conduite.

\section{a) Quel animal dans ces conditions ?}

Les études zootechniques ont d'abord porté en 1972 sur la race locale Lacaune avec des brebis issues du troupeau laitier du domaine. Dès 1974, la race prolifique Romanov, élevée dans les steppes du Nord de la Russie pour sa productivité numérique et pour les qualités de protection thermique de la laine des agneaux d'un mois, a été introduite dans le dispositif (Bouix et al 1985).

Dans la continuité des recherches sur les brebis Lacaune, Romanov et croisées, un protocole diallèle entre ces deux races a été conduit entre 1990 et 1995 pour comprendre les contributions respectives des mères et des agneaux dans la production sur parcours (Bouix et al 2002). Ce protocole classique d'étude de paramètres génétiques entre races, utilisait des béliers de la race spécialisée viande Berrichon du Cher comme testeur des brebis pures ou croisées pour évaluer les effets d'hétérosis maternel.

Le résultat principal dans ce milieu difficile est l'expression des seules qualités maternelles, prolificité comprise, avant le sevrage à 50 jours, à l'ex- 
Tableau 2. Résultats du croisement diallèle entre Lacaune et Romanov (1990-1995). Pour l'ensemble des performances, moyennes ajustées pour l'année et l'âge des brebis. Pour les agneaux, ajustements pour le sexe et le mode de naissance-allaitement.

\begin{tabular}{|c|c|c|c|c|c|c|c|c|c|}
\hline \multirow{2}{*}{$\begin{array}{l}\text { Types génétiques } \\
\text { des agneaux }^{1}\end{array}$} & \multirow{2}{*}{$\begin{array}{c}\text { Prolificité }^{2} \\
(\%)\end{array}$} & \multirow{2}{*}{$\begin{array}{c}\text { Survie } \\
50 \text { jours } \\
\text { (\%) }\end{array}$} & \multirow{2}{*}{$\begin{array}{c}\text { Poids } \\
\text { naissance } \\
\text { (kg) }\end{array}$} & \multicolumn{3}{|c|}{$\begin{array}{c}\text { Croissance } \\
\text { GMQ (g/j) }\end{array}$} & \multicolumn{3}{|c|}{ Qualité des carcasses ${ }^{3}$} \\
\hline & & & & $0-30 \mathrm{j}$ & $\begin{array}{l}30- \\
50 \mathrm{j}\end{array}$ & $\begin{array}{c}70- \\
120 \mathrm{j}\end{array}$ & $\begin{array}{l}\text { Rdt } \\
\text { (\%) }\end{array}$ & $\begin{array}{l}\text { Conf } \\
(1-9)\end{array}$ & $\begin{array}{l}\text { Gras } \\
(\mathrm{mm})\end{array}$ \\
\hline Romanov (R) & 306 & 91,3 & 2,66 & 189 & 164 & 223 & 45,1 & 2,56 & 1,25 \\
\hline Lacaune (L) & 174 & 73,7 & 3,49 & 237 & 208 & 247 & 45,8 & 3,51 & 2,53 \\
\hline Romanov x Lacaune (RL) & 188 & 87,4 & 3,55 & 255 & 229 & 269 & 45,0 & 3,08 & 2,44 \\
\hline Lacaune $\times$ Romanov (LR) & 318 & 91,0 & 2,79 & 192 & 175 & 248 & 45,4 & 3,07 & 2,43 \\
\hline Berrichon du Cher $\times \mathrm{L}$ & 188 & 77,5 & 3,92 & 248 & 234 & 292 & 45,9 & 4,15 & 3,20 \\
\hline Berrichon du Cher x $\mathbf{R}$ & 329 & 86,0 & 2,91 & 197 & 179 & 276 & 46,3 & 4,28 & 3,03 \\
\hline Berrichon du Cher x RL & 249 & 89,0 & 3,40 & 244 & 218 & 284 & 46,0 & 4,33 & 3,05 \\
\hline Berrichon du Cher x LR & 240 & 90,0 & 3,52 & 249 & 218 & 291 & 45,6 & 4,09 & 3,11 \\
\hline
\end{tabular}

1 Pour tout animal issu de croisement, la race du père est en première position et celle de la mère en seconde : agneau $R L=$ père $R$ et mère $L$; agneau Berrichon du Cher $\times \mathrm{RL}=$ père Berrichon du Cher, mère $\mathrm{RL}$ (elle-même de père $\mathrm{R}$ et mère $\mathrm{L}$ ) etc.

2 Prolificité des mères.

3 Rdt : Rendement de la carcasse (poids de carcasse/poids vif),

Conf : Note globale de conformation (système EUROP à 5 classes avec 2 niveaux par classe),

Gras : Epaisseur du gras dorsal mesuré par une fente au-dessus la dernière côte à $3 \mathrm{~cm}$ de l'axe vertébral.

clusion des aptitudes propres des agneaux : avant 50 jours il n'y a par exemple pas de différences de croissance entre les types d'agneaux de mère Romanov, Romanov purs, croisés Lacaune ou Berrichon du Cher (tableau 2). Après le sevrage, les aptitudes directes de croissance mais aussi les qualités bouchères propres aux agneaux peuvent à leur tour s'exprimer en engraissement intensif avec des différences très importantes. Cette expression est toutefois subordonnée à l'obtention d'un développement suffisant de l'agneau sous la mère : la croissance après sevrage des agneaux de mères Lacaune apparaît ainsi relativement faible, qu'il s'agisse d'agneaux de race pure, ou croisés si l'on compare avec les agneaux de mères Romanov à bien moindre potentiel (tableau 2). La survie puis la production requièrent des qualités d'adaptation : protection thermique due aux toisons (Jacquin et al 2002), qualités comportementales des mères à l'agnelage (Boissy et al 2005) puis pendant l'allaitement et aussi capacité des brebis à soutenir un niveau élevé de lactation au moyen de la plasticité des réserves corporelles.

Dans ce sens, en situation d'agnelage autour du $1^{\text {er }}$ avril en plein air et d'exposition aux intempéries, les gènes d'origine Lacaune sont apparus globalement défavorables par rapport aux origines Romanov : taux de survie de 15 à $20 \%$ inférieurs, croissance journalière des agneaux en engraissement guère supérieure à celle des Romanov. Des effets d'hétérosis sont constatés en comparant les différents croisements entre eux et aux races pures : hétérosis sur les effets maternels en comparant parmi les produits de pères Berrichon du Cher les agneaux issus des mères croisées à ceux des mères pures, hétérosis sur les aptitudes des agneaux en comparant les croisés Romanov $\mathrm{x}$ Lacaune aux deux types purs (tableau 2). Des effets d'hétérosis importants se manifestent à la fois sur les aptitudes maternelles avant sevrage et sur les aptitudes directes des agneaux après sevrage sur la croissance.

Tout ceci a conduit à éliminer les brebis de race pure Lacaune, pourtant race locale, mais élevée de longue date en bergerie, aussi bien pour la production de lait que de viande. Les brebis issues des deux croisements réciproques entre les races Romanov et Lacaune ont réalisé la meilleure synthèse en matière de reproduction, de viabilité des jeunes et de croissance des agneaux ; elles ont constitué en outre un bon support de croisement terminal avec les béliers de la race Berrichon du Cher spécialisée pour la viande. Ces animaux croisés possèdent les qualités nécessaires pour un élevage productif sur parcours mais il n'existe malheureusement pas d'organisation de production de brebis croisées Romanov $x$ Lacaune permettant un élevage en croisement à double étage avec des béliers terminaux Berrichon du Cher. Il a été alors décidé de poursuivre les travaux avec un animal voisin des brebis F1 et des agneaux croisés : l'INRA 401 issu d'un métissage entre Romanov et Berrichon du Cher (Tchamitchian et al 1986). L'absorption du troupeau de femelles croisées à l'aide de béliers INRA 401 a été entreprise dans ce but dès la campagne 1999 .

\section{b) La conduite du troupeau}

Les parcs clôturés, d'une quinzaine d'hectares de superficie moyenne, sont pâturés successivement en fonction de leur précocité ou de la capacité de la végétation à être conservée sur pied, pour une utilisation différée. La surface fertilisée couvre 17,7 ha et reçoit $120 \mathrm{~kg}$ d'acide phosphorique/ha tous les trois ans et $65 \mathrm{~kg}$ d'azote/ha chaque année en deux épandages (fin février et fin août). Les clôtures, robustes en périphérie du territoire ou électriques pour les subdivisions intérieures, sont associées à des passages canadiens pour simplifier le travail.

\section{Le cycle zootechnique}

L'agnelage commence vers la fin mars et se termine autour du 20 avril. Il a lieu en plein air dans l'un des parcs, bien exposé, abrité du vent, commode d'accès et facile à surveiller ; la végétation ligneuse y est assez abondante pour offrir aux parturientes protection et lieux d'isolement. Une surface d'environ 2 ha, déplacée chaque année, est délimitée avec un filet électrifié ; les brebis y sont regroupées à partir de la mi-mars au fur et à mesure des prévisions de mise bas. Dès l'agnelage terminé, les brebis allaitantes sont mises à l'herbe dans les parcs fertilisés.

Le sevrage est fixé à la fin juin, après 2 mois $1 / 2$ d'allaitement sur parcours, afin d'obtenir des agneaux bien développés en début d'été, dont l'essentiel de la croissance repose sur la valorisation de la végétation spontanée. Il s'agit aussi de ménager une période d'ap- 
prentissage du pâturage au contact des mères (Thorhallsdottir et al 1990) afin de préparer les agnelles de renouvellement à valoriser le milieu d'élevage. Pour faciliter la séparation, une complémentation modérée en aliment concentré compense la suppression du lait et prépare les agneaux de boucherie à un engraissement rapide après sevrage. L'âge choisi pour l'abattage est de 100 à 120 jours afin de produire des carcasses conformes à la demande dominante du marché en évitant les reports coûteux d'animaux vivants sur l'automne et l'hiver suivants.

Pendant l'été, les agnelles d'élevage sont regroupées dans un troupeau à part maintenu en rotation sur le parcours, en avant des brebis adultes taries.

La mise à la reproduction a lieu en novembre; elle concerne aussi les agnelles les mieux développées (plus de $35 \mathrm{~kg}$ de poids vif) alors âgées de 7 mois, les autres devant attendre l'âge de 18 mois. En fin d'automne et en hiver, pendant la gestation donc, le troupeau continue la rotation sur les parcs de végétation native.

Des procédures spécifiques ont été adoptées pour tenir compte des conditions d'élevage et des aléas qui les caractérisent. Ainsi, le nombre d'agneaux allaités est limité à deux pour les brebis et à un pour les primipares les plus jeunes (âge de 12-13 mois à l'agnelage). Dans le cas de portées plus nombreuses, les agneaux en surnombre sont allaités artificiellement. Les performances attendues et le recours à un génotype prolifique imposent aussi l'usage de compléments alimentaires concentrés à certaines périodes. Pendant le mois précédent l'agnelage, et en tout début d'allaitement, dans un parc d'agnelage avant mise à l'herbe, toutes les femelles gestantes et allaitantes reçoivent un tel complément, la quantité moyenne de $75 \mathrm{~kg}$ variant selon les années en fonction de l'état des animaux. De la même manière, un apport quotidien de 400 g/tête d'aliment concentré a pour but de soutenir la croissance des agnelles en été sur les végétations de report sur pied.

\section{$\underline{\text { Le calendrier d'alimentation }}$}

Le calendrier d'alimentation vise l'adéquation entre la chronologie de la production herbacée primaire et celle des exigences alimentaires des brebis (Jarrige 1979, Thériez et al 1994) en même temps qu'il doit permettre la consommation la plus élevée possible des végétations spontanées. Dès la mise à l'herbe en avril, le troupeau est exclusivement dépendant de l'herbe des parcours, d'abord sur le secteur fertilisé.

Se succèdent ensuite des réserves sur pied pendant tout l'été (120 jours environ), après le tarissement, puis à l'automne, des végétations en repousse dont la quantité disponible est notablement augmentée par la fertilisation. Dès la mi-novembre c'est à nouveau des réserves sur pied qui constituent l'essentiel du fourrage mais des apports d'aliments conservés sont inévitables en plein hiver. Cette alimentation distribuée augmente progressivement jusqu'à devenir exclusive en fin d'hiver : $\mathrm{du}$ foin produit sur l'exploitation comme ration de base et l'aliment concentré de gestation.

Des distributions de foin de qualité moyenne (et/ou de paille) ont également lieu lorsque le fourrage de reports sur pied se raréfie à l'arrivée de l'hiver, ou encore lorsque les intempéries perturbent le pâturage. Il s'agit avant tout d'une mesure de précaution destinée aux individus les plus sensibles au mauvais temps mais qui laisse une part d'autonomie au troupeau. Un foin moyen à médiocre, à l'attrait limité, dissuadera moins les brebis de consommer à nouveau des reports sur pied dès le retour du beau temps. En outre, une éventuelle diversité des préférences individuelles pourra alors mieux s'exprimer.

De la mise à l'herbe en avril et jusque vers la fin janvier, l'apport fourrager est quasi exclusivement représenté par les ressources pastorales, soit près de 300 jours par an, dont une centaine seulement correspond à des périodes de végétation active ; le reste du temps il s'agit de reports sur pied assurant une alimentation qui ne dépasse guère le niveau d'entretien. Un tel calendrier d'alimentation n'a donc pas pour logique d'optimiser à tout instant les apports nutritifs mais bien d'exploiter au maximum l'herbe produite sur le territoire en mettant à profit, à certaines périodes, 1'effet tampon des réserves corporelles des brebis. Avec une productivité individuelle élevée, ces réserves sont susceptibles d'une intense mobilisation pendant la lactation. De plus, les brebis doivent «rustiquer» (Amalbert et Orange 1924) à certaines époques afin de maîtriser le couvert végétal par le biais de niveaux élevés de chargement sur des végétations à des stades avancés. Le calendrier doit donc programmer la reconstitution des réserves corporelles qui peut commencer en été les bonnes années mais dépend surtout des repousses de fin d'été et d'automne, lesquelles ont aussi un effet bénéfique sur la réussite de la lutte. La remise à niveau et les corrections éventuellement nécessaires peuvent se réaliser avec les distributions d'aliments en hiver, notamment la modulation des quantités d'aliments concentrés.

\section{2 / Gérer les ressources pastorales pour la pérennité de l'élevage}

Dès lors que le programme avait atteint le stade du choix d'une seule race à forte prolificité et de modalités d'élevage clairement définies, l'étape suivante était celle de l'optimisation de l'utilisation des ressources pastorales printanières. La conduite du pâturage au printemps devenait cruciale pour réussir l'allaitement sous l'exigence d'un niveau élevé de croissance des agneaux, avec une majorité d'allaitements doubles. La gageure du soutien de la lactation sur les seules ressources spontanées impliquait d'optimiser la combinaison des végétations fertilisées et des végétations natives dans l'alimentation des brebis allaitantes, en gardant à l'esprit que la valorisation durable de la végétation native est au cœur du système car c'est elle qui couvre l'essentiel du territoire.

La question posée était celle de l'aptitude de ces deux types de végétation à répondre aux besoins des brebis allaitantes, avec les meilleures croissances d'agneaux, sur des durées de 70 à 80 jours. La valeur nutritive n'est pas seule en cause ; l'ingestibilité est tout aussi concernée, sachant que ce critère est difficile à évaluer dans ce type de milieu. Il s'agissait alors d'établir des références susceptibles de déboucher sur la mise au point de règles de conduite du pâturage conciliables avec une gestion durable des végétations, et non de réaliser des mesures analytiques sur l'ingestion. De telles références sont en effet nécessaires à la promotion de l'utilisation des ressources, en évitant leur gaspillage et/ou des modes d'exploitation erronés (Chenost et Molénat 1996).

L'option retenue a été celle d'une expérimentation en vraie grandeur, menée pendant les printemps 2000 à 2003 afin de comparer plusieurs modalités d'utilisation des végétations fertilisées et des végétations natives avec un troupeau de brebis (312 à 329 têtes 
selon les années) comportant une forte proportion d'allaitantes (218 à 257 selon les années). Les agneaux allaités, au nombre de 330 à 386 selon les années, avaient un âge moyen à la mise à l'herbe qui avoisinait 3 semaines.

\section{1 / Essayer diverses combinai- sons des végétations fertilisées et des végétations natives}

En début de printemps, seule la végétation fertilisée $\mathbf{F}$ est développée. En mai, la végétation native $\mathbf{N}$ atteint son optimum en quantité et en qualité, alors que $\mathbf{F}$ continue à produire.

Pour alimenter les brebis entre avril et fin juin (sevrage), les profils de production respectifs des végétations $\mathbf{F}$ et $\mathbf{N}$ offrent deux options. La première consiste à exploiter $\mathbf{F}$ au début de l'allaitement puis $\mathbf{N}$ à la fin (option $\mathbf{F}-\mathbf{N}$ ), afin d'octroyer à $\mathbf{N}$ une position stratégique et d'apprécier, dans le temps, son aptitude à satisfaire les besoins des brebis suitées. La seconde option consiste à rester sur $\mathbf{F}$ en deuxième partie d'allaitement (option $\mathbf{F}$ - F) en procédant à des exploitations successives, à la manière de ce qui pourrait se réaliser sur une prairie naturelle de demi montagne. Deux variantes de l'option $\mathbf{F}-$ $\mathbf{N}$, correspondant à deux niveaux d'offre alimentaire sur végétation fertilisée, ont été imaginées, un niveau élevé ${ }_{(\mathbf{H})}$ et un niveau plus restreint $(\mathbf{b})$.

Trois modalités de combinaison des deux types de végétation ont ainsi pu être testées expérimentalement en 2000 et $2001: \mathbf{F}_{(\mathbf{H})}-\mathbf{N}, \mathbf{F}_{(\mathbf{b})}-\mathbf{N}$ et $\mathbf{F}_{(\mathbf{H})}-$ $\mathbf{F}$ (figure 1). Deux périodes peuvent être distinguées, la période 1 exclusivement sur végétation fertilisée $(\mathbf{F})$ et la période 2 où les deux types de végétations sont sollicités, selon les modalités. Le schéma de principe est un pâturage tournant avec 3 lots de brebis d'effectifs comparables (tableau 3) disposant chacun de 4 parcelles clôturées en période 1 et de 3 parcelles en période 2 . Les subdivisions nécessitées par ce schéma étaient réalisées avec des filets électrifiés.

Au cours de la période 1 , deux cycles successifs ont été effectués sur les mêmes parcelles de végétation fertilisée dont la taille était ajustée de telle sorte que le chargement atteigne 17 brebis/ha sur chaque cycle pour $\mathbf{F}_{(\mathbf{H})}$ et 24 brebis/ha pour $\mathbf{F}_{(\mathbf{b})}$. Avec 17 brebis/ha, l'objectif théorique était de prélever $60 \%$ de l'offre, niveau usuel sur de bonnes pelouses. Le chargement de $24 \mathrm{brebis} / \mathrm{ha}$ visait à exploiter plus

Tableau 3. Effectifs d'animaux répartis selon les traitements expérimentaux entre 2000 et 2003.

\begin{tabular}{|c|c|c|c|c|c|c|c|c|}
\hline Année & \multicolumn{2}{|c|}{2000} & \multicolumn{2}{|c|}{2001} & \multicolumn{2}{|c|}{2002} & \multicolumn{2}{|c|}{2003} \\
\hline Modalité & brebis & agneaux & brebis & agneaux & brebis & agneaux & brebis & agneaux \\
\hline$F_{(H)}-N$ & $\begin{array}{l}\text { All. } 73 \\
\text { S. } 37\end{array}$ & 113 & $\begin{array}{l}\text { All. } 86 \\
\text { S. } 20\end{array}$ & 129 & $\begin{array}{ll}\text { All. } 77 \\
\text { S. } 34\end{array}$ & 114 & $\begin{array}{l}\text { All. } 90 \\
\text { S. } 20\end{array}$ & 128 \\
\hline$F_{(b)}-N$ & $\begin{array}{l}\text { All. } 73 \\
\text { S. } 37\end{array}$ & 112 & $\begin{array}{ll}\text { All. } & 85 \\
\text { S. } & 21\end{array}$ & 129 & - & - & - & - \\
\hline$F_{(H)}-F$ & $\begin{array}{ll}\text { All. } 72 \\
\text { S. } 37\end{array}$ & 113 & $\begin{array}{l}\text { All. } 86 \\
\text { S. } 21\end{array}$ & 128 & - & - & - & - \\
\hline$F_{(m)}-N$ & - & - & - & - & $\begin{array}{ll}\text { All. } 67 \\
\text { S. } 28\end{array}$ & 98 & $\begin{array}{ll}\text { All. } 71 \\
\text { S. } 20\end{array}$ & 102 \\
\hline$F_{(H)}-\mathbf{N}, N^{\prime}$ & - & - & - & - & $\begin{array}{ll}\text { All. } 77 \\
\text { S. } 36\end{array}$ & 118 & $\begin{array}{l}\text { All. } 91 \\
\text { S. } 20\end{array}$ & 128 \\
\hline
\end{tabular}

All. = Allaitantes $;$ S. = Antenaises sèches.

complètement la végétation fertilisée au moment de sa forte croissance de printemps.

La période 2 comprenait également deux cycles avec retour sur les mêmes parcelles. En modalité $\mathbf{F}_{(\mathbf{H})}-\mathbf{F}$ le chargement a été maintenu à 17 brebis par ha sur chacun des cycles. Sur végétation native (modalités $\mathbf{F}_{(\mathbf{H})}-\mathbf{N}$ et $\mathbf{F}_{(\mathbf{b})}$ - N), le chargement était de 5 brebis par ha sur chaque cycle et le prélèvement théorique correspondait aussi à $60 \%$ de la production moyenne prévisible. Les changements de parcelles étaient synchrones pour les 3 lots, tous les 4 à 5 jours en période 1 et tous les 6 à 8 jours en période 2 . La mise à l'herbe dans les parcelles expérimentales a eu lieu les 20 et 19 avril et le sevrage les 04 et 02 juillet respectivement en 2000 et 2001 .

En 2002 et 2003, deux nouvelles modalités ont été expérimentées en comparaison avec la modalité $\mathbf{F}_{(\mathbf{H})}-\mathbf{N}$ maintenue comme conduite témoin. D'une part, la modalité $\mathbf{F}_{(\mathbf{b})}-\mathbf{N}$, très contraignante pour l'animal et le végétal, a été remplacée par une modalité $\mathbf{F}_{(\mathbf{m})}-\mathbf{N}$ avec un chargement ramené à 20 brebis par ha en période 1. D'autre part, la modalité $\mathbf{F}_{(\mathbf{H})}-\mathbf{F}$ a été abandonnée de manière a pouvoir tester une modalité appelée $\mathbf{F}_{(\mathbf{H})}-\mathbf{N}$, N' dont la logique était celle d'un pâturage plus libéral améliorant l'offre fourragère en période 2 sur végétation native. $\mathbf{F}_{(\mathbf{H})}-$ $\mathbf{N}, \mathbf{N}^{\prime}$ est identique à $\mathbf{F}_{(\mathbf{H})}-\mathbf{N}$ en période $\mathbf{1}$ et s'en distingue en période $\mathbf{2}$ par l'exploitation successive de deux groupes distincts de 3 parcelles (symbolisés par $\mathbf{N}$ et $\mathbf{N}^{\prime}$ ) préservés de tout pâturage préalable (figure 1). Quand le lot $\mathbf{F}_{(\mathbf{H})}$ $\mathbf{N}$ retournait en juin pour un $2^{\mathrm{e}}$ cycle sur des parcelles qu'il avait déjà exploitées une fois, le lot $\mathbf{F}_{(\mathbf{H})}-\mathbf{N}, \mathbf{N}$ ' poursuivait sur de l'herbe n'ayant pas été pâturée auparavant. Le chargement était identique dans les trois modalités à l'échelle du cycle couvrant la demi période ( 5 brebis/ha), mais sur l'ensemble de la période 2 , il était deux fois moindre dans la modalité $\mathbf{F}_{(\mathbf{H})}-\mathbf{N}, \mathbf{N}^{\prime}$ par rapport aux deux autres modalités ; en outre la nature de l'herbe offerte était différente en $2^{\mathrm{e}}$ moitié de période. Le même schéma de principe comportait 3 lots de brebis d'effectifs comparables en rotation : sur 4 parcelles en période 1 selon 2 cycles puis sur 3 parcelles selon 2 cycles en période 2 sauf pour $\mathbf{F}_{(\mathbf{H})}-\mathbf{N}, \mathbf{N}$ ' qui disposait de 6 parcelles (figure 1 et tableau 3 ). $\mathrm{La}$ mise à l'herbe dans les parcelles expérimentales a eu lieu les 17 et 18 avril et le sevrage le 01 juillet et le 30 juin respectivement en 2002 et 2003.

En fonction des modalités testées, des refus et reports sur pied étaient générés en plus ou moins grande quantité sur les deux types de végétation. Sur les parcelles fertilisées les reports étaient pâturés en juillet par les mêmes lots d'animaux qui y avaient laissé de l'herbe à la fin de la période $\mathbf{1}$. Les lots $\mathbf{F}_{(\mathbf{b})}-\mathbf{N}$ et $\mathbf{F}_{(\mathbf{H})}-\mathbf{F}$, qui avaient totalement exploite leurs parcelles fertilisées, se retrouvaient en juillet sur du parcours natif à un stade avancé de maturité. A partir d'août, les brebis des 3 lots étaient regroupées sur les parcours natifs, notamment les parcelles qui n'avaient été exploitées qu'une fois auparavant, par les agnelles de renouvellement ou par le lot-modalité $\mathbf{F}_{(\mathbf{H})}-\mathbf{N}$, $\mathbf{N}^{\prime}$ en 2002 et 2003.

\section{2 / Production d'herbe et disponibilités fourragères selon les modalités expérimentales}

Les quantités d'herbe présentes sur le terrain ont été mesurées sur chaque parcelle, avant l'entrée de chaque lot 
Figure 1. Représentation schématique des modalités de conduite du pâturage expérimentées au printemps. (les dates de début et fin des périodes ont légèrement varié selon les années; les dates indiquées sont les extrêmes).

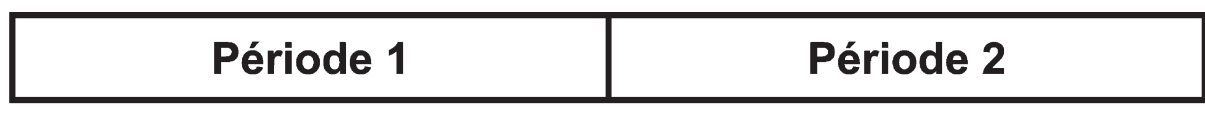

Dates
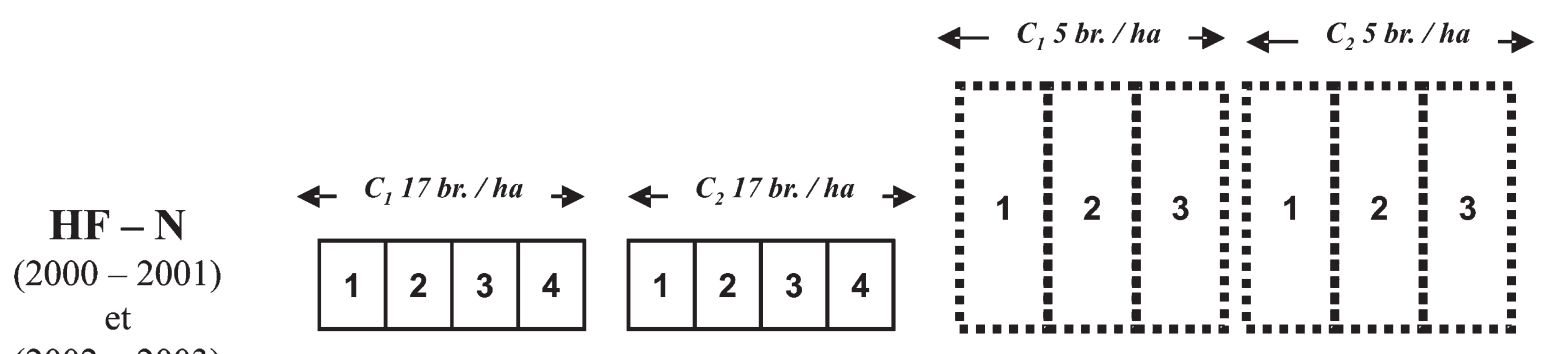

$(2002-2003)$

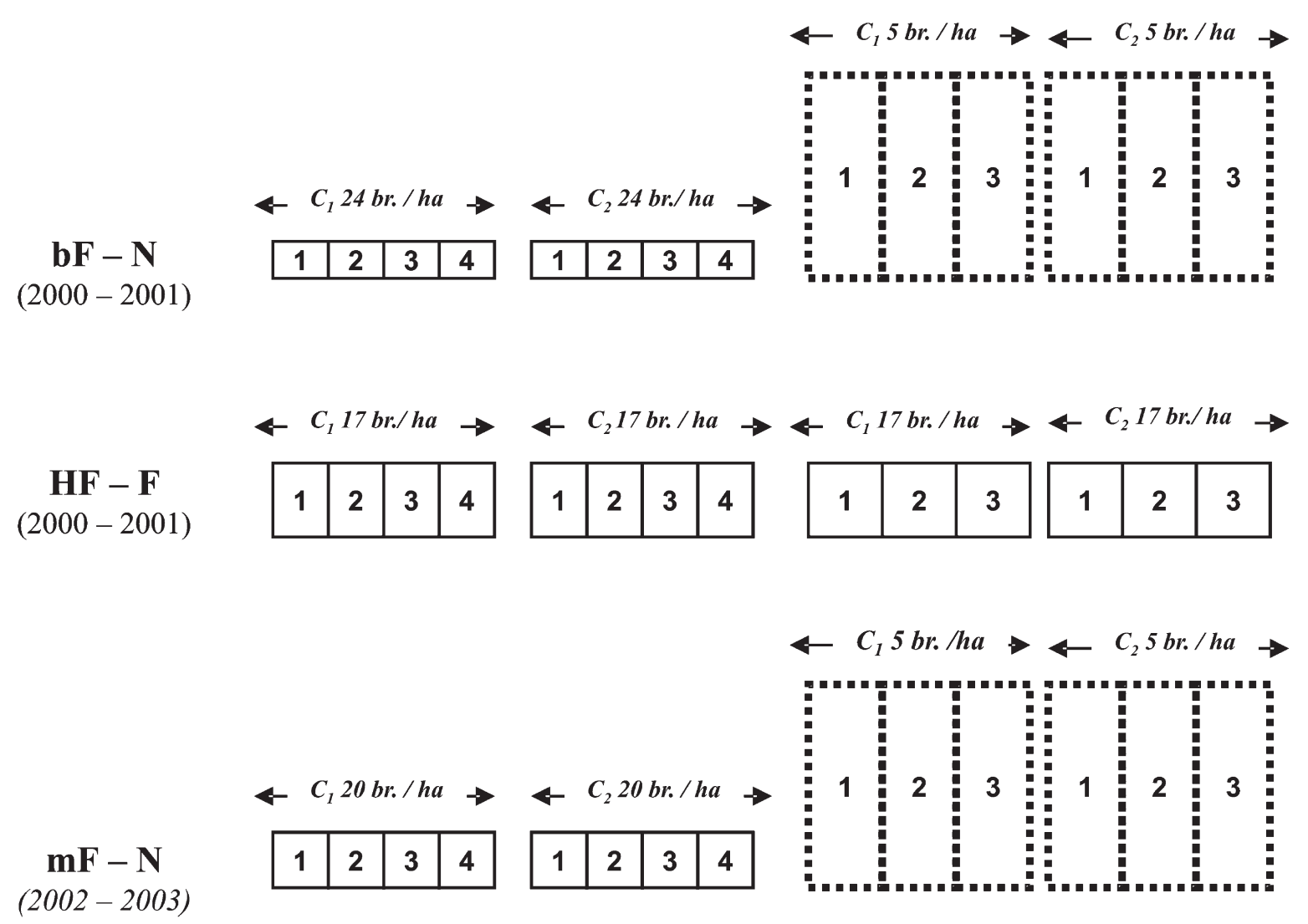

$\mathbf{H F}-\mathbf{N}, \mathbf{N}$ '

$(2002-2003)$

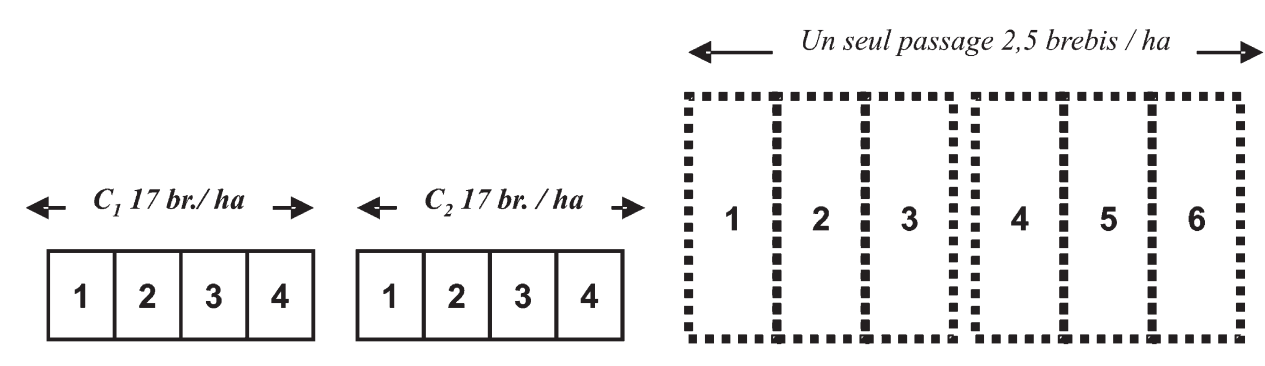

$\square$ Végétation fertilisée

Végétation native 
Tableau 4. Quantités de matière sèche présentes ( $\mathrm{kg} / \mathrm{ha}$ ) et quantités offertes aux brebis (kg / tête / jour) à l'entrée sur les parcelles lors des cycles successifs de pâturage (2000 à 2003).

\begin{tabular}{|c|c|c|c|c|c|c|c|c|}
\hline \multirow{3}{*}{$\begin{array}{l}\text { Traitement } \\
\text { dates indicatives } \\
\text { Année }\end{array}$} & \multicolumn{4}{|c|}{ Quantités présentes (kg MS / ha) } & \multicolumn{4}{|c|}{ Offre fourragère par tête $(\mathrm{kg} \mathrm{MS} / \mathrm{j})^{1}$} \\
\hline & \multicolumn{2}{|c|}{$\begin{array}{c}\text { Période } 1 \\
19 / 04-03 / 05-22 / 05\end{array}$} & \multicolumn{2}{|c|}{$\begin{array}{c}\text { Période 2 } \\
22 / 05-12 / 06-02 / 07\end{array}$} & \multicolumn{2}{|c|}{$\begin{array}{c}\text { Période } 1 \\
19 / 04-03 / 05-22 / 05\end{array}$} & \multicolumn{2}{|c|}{$\begin{array}{c}\text { Période } 2 \\
\text { 22/05-12/06- 02/07 }\end{array}$} \\
\hline & cycle 1 & cycle 2 & cycle 1 & cycle 2 & cycle 1 & cycle 2 & cycle 1 & cycle 2 \\
\hline \multicolumn{9}{|l|}{$\underline{2000}$} \\
\hline $\mathbf{F}_{(\mathrm{H})}-\mathbf{N}$ & 1312 & 1187 & 1238 & 1017 & 4,2 & 5,0 & 12,2 & 10,0 \\
\hline$F_{(b)}-N$ & 896 & 604 & 1238 & 1017 & 2,3 & 1,9 & 12,2 & 10,0 \\
\hline$F_{(H)}-F$ & 1312 & 1187 & 1333 & 1145 & 4,2 & 5,0 & 3,9 & 3,3 \\
\hline \multicolumn{9}{|l|}{$\underline{2001}$} \\
\hline $\mathbf{F}_{(H)}-\mathbf{N}$ & 2258 & 1742 & 1178 & 956 & 8,3 & 8,6 & 11,8 & 10,3 \\
\hline$F_{(b)}-N$ & 1255 & 793 & 1178 & 956 & 3,3 & 2,0 & 11,8 & 10,3 \\
\hline$F_{(H)}-F$ & 2258 & 1742 & 2346 & 2493 & 8,3 & 8,6 & 9,4 & 8,9 \\
\hline \multicolumn{9}{|l|}{$\underline{2002}$} \\
\hline$F_{(H)}-N$ & 1257 & 752 & 1048 & 794 & 4,4 & 3,0 & 11,9 & 9,0 \\
\hline$F_{(m)}-N$ & 1373 & 864 & 1048 & 794 & 4,0 & 2,4 & 11,9 & 9,0 \\
\hline$F_{(H)}-N, N^{\prime}$ & 1257 & 752 & 1338 & 1509 & 4,4 & 3,0 & 12,1 & 16,2 \\
\hline \multicolumn{9}{|l|}{$\underline{2003}$} \\
\hline $\mathbf{F}_{(\mathrm{H})}-\mathbf{N}$ & 1241 & 1013 & 936 & 730 & 4,4 & 3,9 & 10,9 & 8,6 \\
\hline$F_{(m)}-N$ & 1158 & 879 & 936 & 730 & 3,5 & 2,5 & 10,9 & 8,6 \\
\hline $\mathbf{F}_{(\mathrm{H})}-\mathbf{N}, \mathbf{N}^{\prime}$ & 1241 & 1013 & 1248 & 1536 & 4,4 & 3,9 & 13,6 & 16,3 \\
\hline
\end{tabular}

1 L'offre fourragère comprend la quantité présente à l'entrée sur la parcelle augmentée de la croissance de l'herbe pendant la durée du pâturage, estimée par des coupes dans des cages de mise en défens.

d'animaux, par la coupe de 8 placettes de $1 \mathrm{~m}^{2}$. La production des deux types de végétation, constatée au cours des printemps 2000 à 2003 (tableau 4), montre ainsi une variabilité représentative de celle observée sur des durées plus longues (tableau 1).

A même niveau de chargement, les quantités de matière sèche disponibles par tête et par jour ont aussi été très variables d'une année sur l'autre. Le plus souvent aux environs de $4 \mathrm{~kg}$ MS par tête en période 1 sur végétation fertilisée, elles ont été subitement portées à 10 et même plus de $12 \mathrm{~kg}$ MS/tête à l'occasion du transfert sur végétation native en période $\mathbf{2}$, dans les modalités concernées. Les quantités d'herbe présentes au $2^{\mathrm{e}}$ cycle de la période 2 sur les surfaces préservées de pâturage en 2002-2003 ont même atteint le niveau de $16 \mathrm{~kg}$ MS/tête (modalité $\mathbf{F}_{(\mathbf{H})}-\mathbf{N}, \mathbf{N}^{\prime}$ ) alors qu'elles étaient aux alentours de $9 \mathrm{~kg}$ dans les modalités $\mathbf{F}_{(\mathbf{H})}-\mathbf{N}$ et $\mathbf{F}_{(\mathbf{m})}-\mathbf{N}$, sur les parcelles ayant subi une première exploitation.
La valeur nutritive pour l'herbe offerte en Période 1 sur végétation fertilisée a atteint des niveaux élevés comme l'indiquent les analyses fourragères effectuées de 2000 à 2002 (tableau 5). En Période 2 les valeurs étaient plus faibles, sans grande différence entre les deux types de végétation.

\section{3 / Performances animales selon les modalités de gestion des ressources}

Chaque année les lots de brebis, constitués à l'agnelage, étaient équilibrés sur les facteurs âge, poids vif et date de mise bas ainsi que sur la taille de portée, le sexe et le poids de nais-

Tableau 5. Teneur en matière azotée totale et valeur énergétique calculée de l'herbe offerte à l'entrée sur les parcelles en début de périodes, et à mi-période 2 dans le cas de la végétation native.

\begin{tabular}{|l|c|c|c|c|c|c|}
\hline \multirow{2}{*}{ Année } & \multirow{2}{*}{ Végétation fertilisée } & \multicolumn{5}{|c|}{ Végétation native } \\
\cline { 3 - 7 } & & \multicolumn{7}{|c|}{ modalité $\mathbf{F}_{(\mathbf{H})}-\mathbf{N}$} & \multicolumn{2}{c|}{ modalité $\mathbf{F}_{(\mathbf{H})}-\mathbf{N}, \mathbf{N}$ ' } \\
\hline & Période 1 & $\mathbf{P} 2\left(\mathbf{F}_{(\mathbf{H})}-\mathbf{F}\right)$ & début P 2 & mi-P 2 & début P 2 & mi-P 2 \\
\hline & \multicolumn{7}{|c|}{ Matières Azotées Totales (g / kg MS) } \\
\hline 2000 & 207 & 157 & 122 & 113 & - & - \\
\hline 2001 & 175 & 122 & 123 & 112 & - & - \\
\hline 2002 & 216 & - & 122 & 103 & 106 & 105 \\
\hline \multicolumn{7}{|c|}{ Valeur Energétique Calculée (UFL / kg MS) } \\
\hline 2000 & 0,94 & 0,89 & 0,82 & 0,80 & - & - \\
\hline 2001 & 0,94 & 0,81 & 0,83 & 0,80 & - & - \\
\hline 2002 & 0,99 & - & 0,80 & 0,76 & 0,74 & 0,78 \\
\hline
\end{tabular}


sance des agneaux allaités. Les lots comportaient des femelles allaitantes et lutte à 18 mois. Seules les variations de poids des brebis allaitantes et les croissances de leurs agneaux, dont les 2/3 étaient allaités doubles, sont considérées dans ce paragraphe.

Les performances animales ont fait l'objet d'une analyse de variance avec la procédure GLM de SAS 8.2 (SAS Institute Inc., Cary, NC, USA) de façon à estimer sans biais les effets des modalités expérimentales de combinaison des ressources pâturées $\mathbf{F}$ et $\mathbf{N}$. Les valeurs présentées sont les moyennes ajustées et les résultats des tests issus de cette analyse. Sont considérés les facteurs campagne, traitement, taille de portée allaitée (et leurs interactions) et l'âge des brebis ; dans le cas des croissances d'agneaux, sont considérés en plus, le sexe et l'âge au sevrage.

\section{a) Croissance des agneaux}

En début d'allaitement sur végétation fertilisée, avec le chargement de 17 brebis/ha $\left(\mathbf{F}_{(\mathbf{H})}\right.$, Période $\left.\mathbf{1}\right)$, les gains de poids moyens des agneaux ont fluctué selon les années entre 268 et $295 \mathrm{~g} / \mathrm{j}$ (tableau 6). Un chargement plus élevé s'est traduit par une baisse de croissance à peine sensible à $20 \mathrm{brebis} / \mathrm{ha}\left(\mathbf{F}_{(\mathbf{m})}\right.$, et significative à $24 \mathrm{brebis} / \mathrm{ha}\left(\mathbf{F}_{(\mathbf{b})}\right.$, 2000-2001).

Ces performances sont excellentes si on les rapproche de celles obtenues en race INRA 401 en 2003 et 2004 au domaine INRA de La Sapinière en éledes antenaises sèches prévues pour la 2002-2003) mais avoisinant les $20 \mathrm{~g} / \mathrm{j}$

vage intensif de bergerie : $330 \mathrm{~g} / \mathrm{j}$ pour les agneaux allaités simples et $255 \mathrm{~g} / \mathrm{j}$ pour les doubles. La comparaison est possible aussi avec le contrôle national de performances de la campagne 2004 dans les élevages de sélection : 305 et $255 \mathrm{~g} / \mathrm{j}$ respectivement pour les simples et doubles en race Ile-de-France, 270 et $235 \mathrm{~g} / \mathrm{j}$ en race Lacaune, 250 et $205 \mathrm{~g} / \mathrm{j}$ en race des Causses du Lot (Griffon et al 2005).

Un ralentissement des croissances, d'amplitude variable selon les années, s'est toujours manifesté ensuite en $2^{\mathrm{e}}$ moitié d'allaitement. A cet égard, le maintien sur végétation fertilisée s'est révélé particulièrement défavorable (cas de la modalité $\mathbf{F}_{(\mathbf{H})}-\mathbf{F}$ en 2000 et 2001). En revanche, sur la végétation native, avec le chargement adopté en période $\mathbf{2}$, les gains de poids ont moins diminué et nous avons constaté un avantage pour le lot ayant disposé lors du $2^{\mathrm{e}}$ cycle de surfaces préservées jusque là (modalité $\mathbf{F}_{(\mathbf{H})}-\mathbf{N}, \mathbf{N}$ ' en 2002 et 2003). Cette pratique a ainsi permis les profils de croissance d'agneaux les plus réguliers, notamment en 2003, année très sèche dès le mois de mai.

Les poids au sevrage sont le résultat cumulé des effets décrits ci dessus. Par rapport à la modalité $\mathbf{F}_{(\mathbf{H})}-\mathbf{N}$, les augmentations de chargement sur végétation fertilisée en période $\mathbf{1}$ ne les ont affectés que modérément alors que les conditions de pâturage en période 2 ont engendré des écarts importants. Retenons que le retour sur les mêmes surfaces à la fin de mai et dans le courant de juin a entraîné une tendance à la

Tableau 6. Poids au sevrage et gains de poids des agneaux pendant l'allaitement : moyennes ajustées de l'interaction Année x Traitement.

\begin{tabular}{|c|c|c|c|c|c|}
\hline \multirow[b]{2}{*}{ Année } & \multirow[b]{2}{*}{ Traitement } & \multicolumn{3}{|c|}{ Gain Moyen Quotidien de poids vif (g/jour) } & \multirow{2}{*}{$\begin{array}{c}\text { P Sevrage } \\
(\mathbf{k g})\end{array}$} \\
\hline & & Période 1 & $\begin{array}{c}\text { début Période } 2 \\
\text { Cycle } 1\end{array}$ & $\begin{array}{c}\text { fin Période } 2 \\
\text { Cycle } 2\end{array}$ & \\
\hline 2000 & $\begin{array}{l}F_{(H)}-N \\
F_{(b)}-N \\
F_{(H)}-F \\
\end{array}$ & $\begin{array}{l}293 b \\
268 a \\
289 b\end{array}$ & $\begin{array}{c}196 b \\
201 b \\
91 a \\
\end{array}$ & $\begin{array}{l}219 b \\
222 b \\
199 a\end{array}$ & $\begin{array}{l}27,0 \mathrm{c} \\
26,1 \mathrm{~b} \\
24,2 \mathrm{a} \\
\end{array}$ \\
\hline 2001 & $\begin{array}{l}F_{(H)}-N \\
F_{(b)}-N \\
F_{(H)}-F\end{array}$ & $\begin{array}{l}271 b \\
254 a \\
271 b\end{array}$ & $\begin{array}{l}241 b \\
230 b \\
137 a\end{array}$ & $\begin{array}{l}220 b \\
236 b \\
167 a\end{array}$ & $\begin{array}{l}26,7 b \\
26,0 b \\
23,2 a\end{array}$ \\
\hline 2002 & $\begin{array}{l}F_{(H)}-N \\
F_{(m)}-N \\
F_{(H)}-N, N^{\prime}\end{array}$ & $\begin{array}{l}268 \\
266 \\
274 \\
\end{array}$ & $\begin{array}{c}252 b \\
234 a \\
246 a b \\
\end{array}$ & $\begin{array}{l}209 a \\
202 a \\
253 b\end{array}$ & $\begin{array}{l}26,7 a \\
26,0 a \\
28,0 b\end{array}$ \\
\hline 2003 & $\begin{array}{l}F_{(H)}-N \\
F_{(m)}-N \\
F_{(H)}-N, N^{\prime}\end{array}$ & $\begin{array}{l}295 \\
288 \\
295 \\
\end{array}$ & $\begin{array}{l}249 b \\
220 a \\
238 b\end{array}$ & $\begin{array}{l}189 b \\
164 a \\
236 c\end{array}$ & $\begin{array}{l}27,6 \mathrm{~b} \\
26,0 \mathrm{a} \\
28,0 \mathrm{~b}\end{array}$ \\
\hline
\end{tabular}

Les tests sont réalisés intra année et les résultats représentés par des lettres ; les moyennes affectées de deux lettres différentes sont statistiquement différentes au seuil de probabilité $\mathrm{P}=5 \%$. réduction des poids au sevrage, ce qui a été très net dans le cas d'une $3^{\mathrm{e}}$ puis d'une $4^{\mathrm{e}}$ exploitation de la végétation fertilisée (retard de 3 à $4 \mathrm{~kg}$ ). Retenons encore que la liaison avec la quantité de matière sèche offerte n'est pas là clairement apparente ; d'autres caractéristiques du fourrage offert peuvent être en cause comme par exemple les stades végétatifs, susceptibles de décalages d'une année sur l'autre en fonction des conditions météorologiques, avec des effets rapides sur la valeur alimentaire.

\section{b) Variations de poids des brebis}

Les pertes de poids des brebis pendant l'allaitement sont la résultante d'effets annuels aléatoires et d'effets très marqués liés à la disponibilité de l'herbe (tableau 7). En période 1, l'influence de la forte restriction correspondant à la modalité $\mathbf{F}_{(\mathbf{b})}-\mathbf{N}$ a été significative (2000 et 2001). En période 2 , un $3^{\mathrm{e}}$ puis un $4^{\mathrm{e}}$ passage sur végétation fertilisée ont, comme pour les agneaux, aggravé les pertes de poids $\left(\right.$ modalité $\left.\mathbf{F}_{(\mathbf{H})}-\mathbf{F}\right)$. En revanche, sur végétation native en période 2 , les brebis de la modalité $\mathbf{F}_{(\mathbf{b})}-\mathbf{N}$ ont compensé les fortes pertes enregistrées en période 1, leur poids vif moyen au tarissement se rapprochant de celui de la modalité $\mathbf{F}_{(\mathbf{H})}-\mathbf{N}$. De manière similaire, le recours en fin de période 2 à des surfaces préservées jusque là (modalité $\mathbf{F}_{(\mathbf{H})}-\mathbf{N}, \mathbf{N}^{\prime}$ ) a réduit la perte de poids. Les poids des brebis montrent des tendances semblables à celles observées avec les agneaux mais, s'agissant de ruminants, une influence des contenus digestifs n'est pas à exclure suite aux variations importantes des disponibilités fourragères et à d'éventuelles différences de fibrosité.

En été, après tarissement, les différences générées par les modalités du pâturage printanier se sont estompées et toutes les brebis ont retrouvé des poids vifs moyens très voisins.

Les poids des brebis, 57 à $63 \mathrm{~kg}$ à l'entrée sur les parcelles fertilisées selon les années et environ $55 \mathrm{~kg}$ en été, peuvent paraître faibles si on les rapproche de ceux des brebis INRA 401 lors de la mise en lutte au domaine INRA de la Sapinière, en bergerie intensive : $67 \mathrm{~kg}$ en 2003 et $64 \mathrm{~kg}$ en 2004. Cette comparaison est cependant partiellement biaisée par l'absorption encore incomplète en INRA 401 et surtout par une répartition des classes d'âges différente à La Fage : $42 \%$ d'agnelles primipares en raison de la politique d'absorption rapide. Si l'on considère les adultes seules, leur poids 
Tableau 7. Evolution du poids vif des brebis allaitantes. Influence du type de végétation pâturée et de la modalité de pâturage pendant la phase d'allaitement : moyennes ajustées de l'interaction Année x Traitement.

\begin{tabular}{|c|c|c|c|c|c|c|c|c|}
\hline \multirow{2}{*}{ Année } & \multirow{2}{*}{ Traitement } & \multicolumn{3}{|c|}{ Variations de Poids (kg) } & \multicolumn{4}{|c|}{ Poids (kg) } \\
\hline & & Période1 & Période2 & été & début P1 & fin P1 & fin P2 & été \\
\hline 2000 & $\begin{array}{l}F_{(\mathrm{H})}-\mathbf{N} \\
\mathbf{F}_{(\mathrm{b})}-\mathbf{N} \\
\mathbf{F}_{(\mathrm{H})}-\mathbf{F}\end{array}$ & $\begin{array}{l}-5,0 b \\
-6,8 a \\
-5,3 b\end{array}$ & $\begin{array}{r}-6,6 c \\
-5,6 b \\
-10,2 a \\
\end{array}$ & $\begin{array}{l}3,3 b \\
3,2 b \\
5,8 a\end{array}$ & $\begin{array}{l}63,2 \\
63,0 \\
63,1 \\
\end{array}$ & $\begin{array}{l}58,2 \\
56,2 \\
57,8\end{array}$ & $\begin{array}{l}51,6 \\
50,8 \\
47,6 \\
\end{array}$ & $\begin{array}{l}55,0 \\
54,0 \\
53,4\end{array}$ \\
\hline 2001 & $\begin{array}{l}\mathbf{F}_{(\mathrm{H})}-\mathbf{N} \\
\mathbf{F}_{(\mathrm{b})}-\mathbf{N} \\
\mathbf{F}_{(\mathrm{H})}-\mathbf{F}\end{array}$ & $\begin{array}{l}-1,5 b \\
-7,0 a \\
-2,0 b\end{array}$ & $\begin{array}{l}-6,3 b \\
-1,5 c \\
-9,7 a\end{array}$ & $\begin{array}{l}1,7 \mathrm{~b} \\
2,0 \mathrm{~b} \\
3,1 \mathrm{a}\end{array}$ & $\begin{array}{l}62,4 \\
62,4 \\
63,2 \\
\end{array}$ & $\begin{array}{l}60,9 \\
55,4 \\
61,1\end{array}$ & $\begin{array}{l}54,6 \\
53,9 \\
51,4\end{array}$ & $\begin{array}{l}56,3 \\
55,9 \\
54,6\end{array}$ \\
\hline 2002 & $\begin{array}{l}F_{(H)}-N \\
F_{(m)}-N \\
F_{(H)}-N, N\end{array}$ & $\begin{array}{l}-2,2 \\
-2,1 \\
-2,8\end{array}$ & $\begin{array}{l}-4,9 b \\
-5,7 a \\
-4,1 c\end{array}$ & $\begin{array}{l}0,6 \mathrm{~b} \\
3,4 \mathrm{a} \\
0,4 \mathrm{~b}\end{array}$ & $\begin{array}{l}60,7 \\
60,4 \\
61,2 \\
\end{array}$ & $\begin{array}{l}58,5 \\
58,3 \\
58,4\end{array}$ & $\begin{array}{l}53,6 \\
52,6 \\
54,3\end{array}$ & $\begin{array}{l}54,2 \\
55,9 \\
54,8\end{array}$ \\
\hline 2003 & $\begin{array}{l}F_{(H)}-N \\
F_{(m)}-N \\
F_{(H)}-N, N\end{array}$ & $\begin{array}{l}2,9 \\
3,1 \\
2,2\end{array}$ & $\begin{array}{l}-8,1 a \\
-8,4 a \\
-7,0 b\end{array}$ & $\begin{array}{l}1,6 \mathrm{~b} \\
2,4 \mathrm{a} \\
1,1 \mathrm{~b}\end{array}$ & $\begin{array}{l}57,2 \\
57,5 \\
58,8\end{array}$ & $\begin{array}{l}60,1 \\
60,7 \\
61,1\end{array}$ & $\begin{array}{l}52,0 \\
52,3 \\
54,1\end{array}$ & $\begin{array}{l}53,5 \\
54,7 \\
55,2\end{array}$ \\
\hline
\end{tabular}

Les tests sont réalisés intra année et les résultats représentés par des lettres; les moyennes affectées de deux lettres différentes sont statistiquement différentes au seuil de probabilité $\mathrm{P}=5 \%$.

moyen en été se situe aux alentours de $63 \mathrm{~kg}$, inférieur d'environ $10 \%$ à celui des INRA 401 de La Sapinière. C'est la conséquence à la fois du milieu difficile et du choix technique consistant à faire exploiter au maximum les végétations spontanées en demandant un effort important aux animaux.

\section{4 / Interprétation des résultats dans une logique de valorisation des parcours}

En début d'allaitement, les modalités réduisant les disponibilités fourragères sur une végétation fertilisée active n'ont eu, sur la croissance des agneaux, que des conséquences limitées; les réserves corporelles des brebis semblent avoir joué un rôle tampon pour des agneaux alors quasi exclusivement dépendants du lait maternel. En deuxième partie d'allaitement, les différences dans l'offre fourragère ont eu, en revanche, des effets très marqués qui suscitent des interrogations relatives aux quantités mais aussi aux stades végétatifs et aux conditions du pâturage. Une $3^{\mathrm{e}}$ et une $4^{\mathrm{e}}$ exploitation de la végétation fertilisée étaient très préjudiciables aux performances animales alors qu'un pâturage des végétations natives était plus favorable, surtout si l'herbe était nouvelle, préservée, à chaque changement.

Avec l'avancement de la lactation, la production laitière des mères baisse et les agneaux mangent de plus en plus d'herbe. Passée la mi-mai, l'herbe disponible doit alors répondre à un double besoin, d'une part éviter la diminution trop rapide des quantités de lait produites (notamment pour les dernières brebis agnelées) et d'autre part motiver les agneaux pour le pâturage en leur permettant de compenser la baisse de disponibilité du lait maternel. Les agneaux et leurs mères se trouvent donc en concurrence pour les fractions les plus attractives de l'herbe et aussi pour les plus digestibles et les plus riches en nutriments. Dans ces circonstances, les choix alimentaires dont on sait depuis longtemps qu'ils s'expriment avec force dans ce milieu (Molénat et al 1975, Leclerc et al 1978) pourraient avoir un rôle d'autant plus grand que les performances animales attendues sont élevées. Comme les plantes et les organes les plus intéressants sont, selon toute probabilité, consommés en priorité lors de l'entrée sur une nouvelle parcelle (O'Reagain et Schwartz 1995), la disparition des meilleures fractions de la biomasse fourragère peut être cause de régression rapide de la qualité de l'herbe dans le cas d'une offre limitée. En outre, sur ces pelouses steppiques, l'attractivité et la valeur nutritive des espèces végétales peuvent souffrir des stades végétatifs brefs en conséquence de cycles rapides.

L'exigence d'un niveau élevé de performance zootechnique met ainsi l'accent sur la manière dont la gestion des végétations natives peut limiter les effets de la concurrence pour l'herbe entre les agneaux et leurs mères. La traduction en terme de pratiques et de procédures suggère une grande attention à la fréquence des changements de parcelle (dès que les meilleures fractions de la biomasse fourragère auront été prélevées) et à l'intérêt qu'il peut y avoir à offrir de l'herbe nouvelle à chaque changement de parcelle afin de tirer le meilleur parti de la diversité spécifique de ces végétations.
Une autre question que 1'on peut se poser est celle des seuils de quantités disponibles en relation avec le type de végétation et son état d'avancement qui sont fonction notamment du substrat et de l'éventuelle fertilisation. En première approche, dans nos conditions et compte tenu des performances attendues, les ordres de grandeur semblent être aux alentours de $4 \mathrm{~kg}$ de MS/brebis/jour au début du printemps sur végétation fertilisée et de plus de $10 \mathrm{~kg}$ à la fin sur végétation native.

\section{3 / En perspective : des cohérences pour un élevage ovin viande performant et durable sur parcours}

L'étape ayant consisté à préciser les règles d'usage de la phytomasse pastorale au printemps ouvre de solides perspectives pour un élevage ovin viande performant sur parcours. Ces nouveaux acquis doivent maintenant prendre leur place par rapport aux autres éléments du système, de manière à exprimer sa cohérence et à en dégager les aspects innovants.

L'assemblage des éléments concrétise un exemple de système de production expérimental (peut-on parler de prototype ?) dont la cohérence dépend d'abord de la réalisation d'un bon compromis entre les objectifs de production animale et les objectifs d'entretien du milieu (Molénat et al 1976). La convergence des procédures de conduite du troupeau par rapport aux objectifs retenus est restée l'élément fédérateur de l'équipe pluridisciplinaire qui s'est attachée à adapter les connexions internes au système de manière à atteindre la meilleure compatibilité entre elles et à conférer à l'ensemble un maximum de cohésion. Le système technique doit aussi rester connecté avec des préoccupations plus économiques et sociales, au premier rang desquelles nous avons cité les charges de structure (bâtiments, clôtures,...) et le travail (volume, pénibilité, répartition,...). Un examen détaillé des différentes connexions concernant la conduite d'élevage et l'impact sur le milieu, permettra maintenant de percevoir, dans sa globalité, un exemple de fonctionnement de troupeau ovin viande prolifique sur parcours ; il permettra aussi d'abonder les démarches prospectives de l'ensemble des acteurs concernés sur la durabilité d'un tel élevage. 


\section{1 / Cohérence d'un système de production animale}

La philosophie générale du programme de recherche fait référence à la condition préalable consistant à lier l'avenir des territoires pastoraux à l'élevage, ce qui revient à le lier aussi à l'avenir des éleveurs, lui-même très dépendant de la qualité du cheptel et de ses productions. Seul un cheptel en bon état (sanitaire, nutritionnel, etc.) pourra en effet garantir à la fois la mâ̂trise de l'impact animal sur le milieu et la rémunération des activités d'élevage, avec en conséquence un soutien actif de la motivation de l'éleveur (Rouquette et al 1997).

Pour l'élaboration du système de production expérimental, trois questions globales étaient posées, celle de l'animal productif adapté, celle de la chronologie de la production herbacée primaire face aux exigences alimentaires du cycle zootechnique et celle des exigences de protection du milieu et des ressources (indissociables des exigences de production dans les territoires pastoraux : Molénat et al 1999, Molénat et al 2003). Des solutions se sont dégagées par le biais de recherches génétiques originales et d'une approche volontariste du rôle de la diversité des végétations à propos de leur fonction pastorale comme de leur fonction écologique.

\section{a) Le choix d'un type d'animal adapté}

Le type d'animal utilisé, 1/2 sang Romanov, a largement contribué à la réussite du projet. Les brebis INRA 401, à la suite des croisées Romanov $\mathrm{x}$ Lacaune, semblent capables de réaliser une synthèse entre des qualités d'adaptation nécessaires pour explorer l'espace et valoriser les végétations d'une part, et des aptitudes de production susceptibles de générer un revenu significatif pour l'éleveur d'autre part. Les qualités d'adaptation sont d'abord la protection thermique de l'agneau nouveau-né et la rapidité de l'établissement du lien mère-jeune en dehors de toute surveillance humaine, lors de l'agnelage en plein air en tout début de printemps à 800m d'altitude (Bouix 1992). L'autonomie manifeste de ce type d'animal pour l'exploration et la valorisation des parcours, ainsi que sa sociabilité (Boissy et al 2005) participent à la bonne valorisation du milieu et à la transmission de cette aptitude par apprentissage entre générations. Des pratiques d'élevage spécifiques, mises en œuvre au moment de l'agnelage et lors du sevrage des agnelles, ont créé puis entretenu une relation à l'homme nécessaire lors des rares manipulations d'un tel troupeau qui vit en liberté dans les parcelles (Boissy et al 2001).

Les premiers résultats de production sont encourageants et comparables à ceux des croisés Romanov x Lacaune pour la productivité numérique ; il en est de même pour la croissance dans le cas des lots expérimentaux soumis aux modalités de pâturage les plus favorables. Ce recul de quatre campagnes successives est encore limité et l'appréciation doit être nuancée dans la mesure où ces quatre campagnes correspondaient à la phase d'absorption de l'ancien troupeau de brebis F1 par des béliers INRA 401 avec une relative hétérogénéité du génotype d'animaux, cependant tous $1 / 2$ sang Romanov, et une forte proportion de primipares (liée au souhait d'une absorption rapide). Avec des taux d'absorption supérieurs, les résultats des animaux de 2004 et 2005 ont confirmé ces premières indications.

Alors que la vocation première de la race INRA 401 la destinait à augmenter la productivité de formes d'élevage intensives, le pari non dénué de risques de son introduction dans un tel système d'élevage en plein air intégral, dans un milieu aussi contraignant que le Causse du Larzac, apparaît comme un choix judicieux. Cette race s'est révélée à l'usage capable de tirer profit de milieux pastoraux divers (Lecomte et al 1996) ; elle possède vraisemblablement des aptitudes adaptatives concernant l'alimentation et la nutrition permettant de gérer ses réserves corporelles au bénéfice de la production zootechnique. Les taux élevés de reproduction et de survie sont le signe de l'adaptation des animaux au milieu, de l'état de bien-être et de qualité du cheptel, à la fois cause et conséquence de la réussite dans ce milieu difficile.

b) Adéquation entre la production primaire et les exigences alimentaires du cycle zootechnique

Un des fondements du système réside dans la fertilisation de portions de parcours en conjonction avec un agnelage de tout début du printemps. Les agneaux, allaités doubles pour les $2 / 3$, sont élevés exclusivement sur parcours pendant la plus grande partie de l'allaitement (mi-avril à fin juin), avec des croissances supérieures à $250 \mathrm{~g} / \mathrm{j}$ en moyenne sous l'effet d'une bonne gestion des végétations (les fertilisées et les natives). Par la rapidité de la rotation et la modulation des chargements, mais aussi par la présence permanente sur le pâturage, les animaux sont mis en situation de tirer profit de la diversité des espèces végétales et de leurs décalages phénologiques dans le temps. En outre, l'augmentation de la production d'herbe en avril permet de préserver des parcelles natives pour la fin d'allaitement, ce qui accroît la sécurité des apports alimentaires, en particulier face au risque de sécheresse de fin de printemps.

La volonté de permettre aux agnelles de renouvellement d'apprendre au contact des mères à bien valoriser la végétation des parcours a allongé l'allaitement. Ce mode de conduite s'est soldé par un fléchissement des gains de poids des agneaux après la mi-mai, conséquence somme toute logique en milieu pastoral. Les croissances des agnelles restant toutefois supérieures à $200 \mathrm{~g} / \mathrm{j}$ pendant cette période, leur avenir n'est pas pénalisé. Chaque automne, 55 à $65 \%$ d'entre elles peuvent même être mises à la reproduction à l'âge de 7 mois moyennant une conduite adaptée après sevrage.

Les brebis valorisent bien les végétations pastorales et doivent mobiliser suffisamment leurs réserves au profit des agneaux qu'elles allaitent. Pour reconstituer leurs réserves, les brebis valorisent au maximum les refus et reports sur pied, en été et en hiver ; elles peuvent également saisir des opportunités comme des repousses de prés de fauche impossibles à récolter en automne. Pendant tout l'automne, elles consomment principalement des repousses de végétations pastorales (1000 kg MS/ha sur parcelles fertilisées et $200 \mathrm{~kg} \mathrm{MS} / \mathrm{ha}$ ailleurs) qui contribuent à une bonne préparation à la lutte.

\section{c) L'élevage des jeunes}

Avant toute autre considération, le taux de survie des agneaux est une condition de l'avenir de ce type d'élevage ovin prolifique en plein air intégral à $800 \mathrm{~m}$ d'altitude. A cet égard, si l'on se réfère aux années 2000 à 2003, la mortalité des agneaux jusqu'au sevrage a été de $16 \%$ (tableau 8); elle est comparable à celle observée sur des brebis élevées en bergerie avec la même prolificité de 2,3 agneaux par tête. Cette prolificité est vraisemblablement trop élevée pour ces conditions d'élevage, probablement en conséquence de la pratique de l'insémination artificielle imposée par des nécessités génétiques et sanitaires dans une phase de constitution du troupeau expérimen- 
Tableau 8. Taux de mortalité des agneaux allaités sur parcours et proportions d'agneaux allaités artificiellement au cours des années 2000 à 2003.

\begin{tabular}{|c|c|c|c|c|}
\hline \multirow{2}{*}{ Année } & \multirow{2}{*}{$\begin{array}{c}\text { Prolificité } \\
\text { (agneaux/mère) }\end{array}$} & Allaitement & \multicolumn{2}{|c|}{ Mortalité des agneaux (\%) } \\
\cline { 4 - 5 } & artificiel (\%) & néonatale (<48h) & postnatale (>48h) \\
\hline 2000 & 2,24 & 23,8 & 10,4 & 5,8 \\
\hline 2001 & 2,31 & 26,4 & 9,9 & 8,0 \\
\hline 2002 & 2,36 & 32,2 & 5,7 & 10,3 \\
\hline 2003 & 2,29 & 27,6 & 8,2 & 6,0 \\
\hline
\end{tabular}

tal. En routine et avec une lutte libre, la prolificité devrait pouvoir être ramenée vers 1,8 à 1,9 agneaux nés par brebis et le taux de mortalité vers $12 \%$, réduisant aussi les contraintes d'allaitement artificiel qui ne concerneraient plus que $15 \%$ environ des agneaux au lieu de $27,5 \%$.

Les taux de survie satisfaisants sont à porter au crédit des qualités maternelles des brebis INRA 401 ainsi qu'à celui de l'expérience acquise par les hommes dans le contrôle de la prédation. C'est ainsi que le filet électrifié a été utilisé efficacement pendant la période d'agnelage comme protection contre les renards et les chiens errants dont les attaques représentent un frein sérieux à la mise en valeur des zones pastorales défavorisées.

La réussite d'un allaitement plutôt long, accompagné de croissances d'agneaux soutenues, peut être considérée comme un atout du système. L'objectif de valorisation des végétations est en effet atteint et, de plus, les agneaux ont un bon développement au moment du sevrage, ce qui garantit l'avenir des femelles de renouvellement et augure d'une finition rapide des animaux de boucherie. Pour une majorité de ces derniers la finition peut en effet s'effectuer dans le mois qui suit le sevrage en produisant des carcasses conformes à la demande du marché. L'apprentissage du pâturage au contact des mères, voulu d'abord pour les agnelles, est également avantageux dans une perspective d'engraissement et de finition à l'herbe (Prache 1987, Thériez et al 1997). Des études actuellement en cours démontrent la possibilité de finir les agneaux mâles sur des repousses de prés de fauche, avec des quantités d'aliments concentrés de l'ordre de $30 \mathrm{~kg}$ par tête (Biagini et al 2003). Les quantités d'aliments concentrés nécessaires pour l'élevage des jeunes dans l'état actuel du programme, peuvent donc être estimées à une trentaine de $\mathrm{kg}$ par agneau de boucherie pour la finition et à une cinquantaine de $\mathrm{kg}$ par agnelle d'élevage pour la complémentation estivale dans la perspective de mise à la lutte de près des $2 / 3$ d'entre elles à 7 mois.

d) Contributions des ressources alimentaires à la couverture des dépenses énergétiques

Les contributions respectives des ressources à l'alimentation du troupeau ont un rôle primordial dans le programme, au niveau des connexions entre le système de production expérimental et les préoccupations de nature économique, notamment celles qui concernent l'autonomie fourragère.

Avec les taux de consommation de la biomasse herbacée retenus, c'est un effectif de 1'ordre de 350 têtes qui est nécessaire pour exploiter les 280 ha de parcours du domaine de La Fage, soit 0,8 ha de parcours par animal. Dans ces conditions, la volonté de faire en sorte que la croissance des agneaux soit le plus possible issue de l'herbe des parcours a conduit à fertiliser $6 \%$ de la surface totale. Cette proportion tient compte des performances attendues de la part des animaux de race INRA 401 (et serait plus faible avec des races moins performantes). En se référant alors à la modalité $\mathbf{F}_{(\mathbf{H})}-\mathbf{N}, \mathbf{N}$, le calcul sur la base de l'énergie fait ressortir une contribution du parcours à l'alimentation annuelle des animaux d'élevage de $68 \%$, répartie entre la végétation fertilisée $(24 \%)$ et la végétation native $(44 \%)$. Il reste sensiblement $1 / 3$ des dépenses énergétiques à couvrir par des aliments distribués (18\% par le foin et $14 \%$ par du concentré), en conséquence d'hivers relativement rudes sur le Larzac, mais aussi du choix des taux de consommation élevés de la biomasse herbacée dans un souci d'entretien des végétations par des brebis dont le niveau de productivité exige une bonne maîtrise des fluctuations de réserves corporelles. Les quantités annuelles de foin par brebis ou par agnelle, de l'ordre de $150 \mathrm{~kg}$, sont du même ordre que celles observées dans de nombreuses situations d'élevage ovin de demi montagne à vocation pastorale, mais souvent avec des charges de bâtiments et avec des productivités moindres : quantités inférieures à celles constatées sur le Causse Méjean (Groupe de recherches INRA-CNRS 1979) et légèrement supérieures à celles des vallées pyrénéennes (Gibon 1981) où le pâturage hivernal de prairies joue un grand rôle. Pour ce qui concerne l'aliment concentré, les quantités totales ramenées à la brebis (compléments d'élevage et d'engraissement compris) s'établissent à $105 \mathrm{~kg} / \mathrm{an}$, très comparables à celles relatées pour les élevages «extensifs autonomes» du Montmorillonnais (Benoit et al 1997).

Dans l'état actuel, foin inclus, les productions du domaine peuvent contribuer aux dépenses énergétiques des femelles d'élevage à hauteur de $86 \%$. Cette proportion pourrait être augmentée en remplaçant tout ou partie du concentré de gestation par des repousses de prés de fauche en début d'hiver et de l'orge produit sur le domaine pour le mois de mars.

\section{2 / Cohérence d'une gestion des ressources et des végétations}

L'alimentation du troupeau répond à des enjeux de valorisation des ressources locales associés à un ancrage durable du système dans le terroir, dont l'intérêt a été souligné par Roggero et al (1996). Dès lors la stratégie pastorale consiste à organiser les interactions complexes entre les animaux domestiques et l'environnement végétal (végétations, conditions écologiques, fertilité des sols, etc.) (Baumont et al 2000). Il s'agit d'une gestion sur le long terme constituant un mode de pilotage des végétations avec la double fin de renouvellement des ressources alimentaires du troupeau et d'entretien du milieu. Les procédures adoptées doivent mettre les animaux en condition d'exploiter les fractions les plus judicieuses des végétations pastorales selon l'époque dans le cycle zootechnique et dans les cycles végétatifs des espèces pâturées.

Dans ces milieux, la difficulté et le coût élevé du travail mécanique, alliés aux arguments écologiques, militent pour donner à l'animal une place prépondérante dans l'entretien des végétations. Il est alors important d'obtenir à certaines époques la consommation d'un maximum de matériel végétal, y compris des végétaux sénescents, mais également des plantes qui deviendraient indésirables si elles prenaient trop d'extension (chardons par exemple). Il s'agit d'une sorte de remise à niveau de la pelouse qui s'effectue pendant les phases de moindres besoins des 
brebis, en été et en hiver, en jouant sur les chargements et les durées de séjour dans les parcelles. La dégradation des végétations pastorales peut ainsi être évitée, ce qui leur assure un meilleur démarrage, à l'automne pour des repousses de bonne qualité, de même qu'au printemps suivant.

Les chargements instantanés relativement élevés, avec l'aide de points d'eau mobiles et de clôtures électriques amovibles, sont utilisés comme des outils de maîtrise de l'intensité du broutement et de l'homogénéité de l'exploration des surfaces pâturées (Lambert et al 1985). L'objectif peut être atteint avec des parcs dont la superficie et le nombre garantissent une gestion satisfaisante de la végétation, le maintien de l'ouverture du milieu et la préservation de la diversité floristique (Hubert et Grandjanny 1989). Il y a intérêt à ne pas trop agrandir les parcs afin d'en disposer d'un plus grand nombre, ce qui offre l'opportunité de laisser la végétation de certaines parcelles arriver à maturité une année sur trois ou quatre et permet aux espèces de terminer leur cycle, avec reconstitution des banques de semences ou des organes de réserves. Une telle disposition peut représenter une bonne précaution pour le maintien de la biodiversité lors d'accidents climatiques tels que les fortes températures de 2003. Pour un effectif de 200 à 300 brebis, un bon compromis réside dans des parcs de végétation native d'une quinzaine d'hectares chacun, dont la durée de pâturage varie entre 5 et 10 jours selon la saison. Des parcelles plus petites imposeraient un rythme de rotation trop rapide avec des manipulations d'animaux trop fréquentes.

Remarquons encore que le cantonnement du troupeau sur les parcelles fertilisées au début du printemps équivaut de facto à une mise en défens transitoire des autres surfaces. En outre, les végétations natives sont soumises en mai-juin à des taux de prélèvement qui évitent à la fois de surpâturer la pelouse et de pénaliser les animaux.

Ainsi, la diversification des ressources au printemps autorise une plus grande souplesse dans la répartition de l'impact printanier du troupeau sur la plus grande partie du territoire (végétations natives $=94 \%$ de la surface) et facilite la prise en compte des critères écologiques. Les perturbations subies par les végétaux sont mieux maîtrisées et les plus fragiles peuvent terminer leur cycle et assurer leur pérennité. Les dérives dans l'équilibre entre les espèces végétales pouvant être évitées, l'objectif d'entretien du milieu en termes d'ouverture, de proportions entre les espèces végétales et de pérennité pastorale, peut être atteint (Hubert et Alard publication en préparation).

\section{Conclusion}

Un élevage ovin allaitant performant tirant du parcours $68 \%$ de son énergie alimentaire a été possible au domaine de La Fage tout en entretenant la végétation pastorale.

Il s'est appuyé d'une part sur un animal réalisant une bonne synthèse entre des caractères d'adaptation et de production et, d'autre part sur la diversité des ressources pâturées qui a même été artificiellement accrue. Une fertilisation raisonnée de certains secteurs de parcours a ainsi créé de nouvelles ressources qu'une production printanière en avance de 3 à 4 semaines sur la pousse naturelle place dans la position stratégique d'assurer une trentaine de jours d'alimentation de qualité en début d'allaitement. Ici le rôle de complément stratégique ne réside pas seulement dans la stabilisation du système technique face à des risques de pénurie transitoires (Talamucci et Pardini 1996) ; il consiste aussi à lever le blocage lié à un facteur limitant afin d'élargir les perspectives économiques en soutenant la productivité zootechnique.

Pour la cohérence zootechnique du système de production animale, un travail important a été mené sur le calendrier d'exploitation des végétations pâturées. Il aboutit à un enchaînement consistant à commencer au printemps, dès la fin de l'agnelage, par les surfaces fertilisées avant de rejoindre les parcours natifs vers la mi-mai. Jusqu'à la fin juin, un taux de consommation délibérément modéré des végétations privilégie les apports nutritifs aux animaux. Les pratiques de pâturage soutiennent ainsi la production laitière des brebis et limitent la compétition qui s'installe progressivement entre les mères et leurs agneaux pour l'herbe ; elles assurent après sevrage un bon développement aux agnelles sur leurs parcelles réservées.

Les brebis peuvent ensuite être maintenues sur les parcours : en été elles valorisent les refus et reports sur pied générés en grande quantité en maijuin ; en automne elles consomment des repousses pendant la lutte et la reconstitution des réserves corporelles ; en hiver elles retrouvent des reports sur pied.

En sécurisant la phase d'allaitement, qui est celle où la marge de manœuvre alimentaire est la plus faible, la fertilisation a permis de tenir le troupeau sur les parcours. Pour les autres phases zootechniques, moins sensibles, les ressources natives, même mûres peuvent suffire. Dès lors, une exploitation intense des végétations pastorales est possible, en été aussi bien qu'en hiver, afin d'entretenir la pelouse. La présence permanente des troupeaux sur le parcours en stimule la vie biologique par le biais notamment du recyclage des matières organiques. La rotation $\mathrm{du}$ pâturage, associée à des parcs de dimension raisonnée, permet de répartir l'impact sur les végétations en tenant les objectifs de production sans négliger l'entretien du milieu. Des apports modérés de fertilisants sur une superficie très limitée ont ainsi assuré la réussite d'une dynamique de reconquête des parcours par l'élevage, au domaine de La Fage. Le système expérimenté s'inscrit bien dans une logique d'autonomie fourragère basée sur les ressources locales avec un complément d'améliorations pastorales jouant un rôle de catalyseur (Molénat et Chenost 1996). En outre, les végétations sont demeurées ouvertes et leur biodiversité n'a pas été altérée (Hubert et Alard publication en préparation), ce qui respecte les objectifs d'origine de recherche d'une voie agronomique de gestion pastorale peu agressive.

Le système de production élaboré est également largement conditionné à la fois par le type d'animal et par la maîtrise des comportements des animaux à l'égard des végétations. Sur ces points, l'animal exigeant qu'est l'INRA 401 a constitué un bon moyen d'évaluation des végétations pâturées au printemps ainsi que des capacités animales de récupération, au cours de l'été à partir de reports de végétation desséchés, puis ensuite avec des repousses ou des compléments alimentaires hivernaux. En matière alimentaire, les brebis $1 / 2$ sang Romanov semblent donc présenter une plasticité dont les mécanismes mériteraient des recherches spécifiques approfondies. Ces caractères d'adaptation synthétisés d'ordinaire dans le concept qualitatif de rusticité ont participé à la réussite de cet élevage sur parcours. Ils représentent aussi, pour les chercheurs impliqués dans ce travail, des objets de recherche sur d'autres caractères que les performances zoo- 
techniques classiques, de nature à infléchir à terme les objectifs de sélection et les pratiques d'élevage. Concernant la conduite des animaux et du pâturage, il a fallu trouver les ajustements en cohérence avec les objectifs de production animale et d'entretien du milieu. Un pilotage des comportements a été mis en place de manière à stimuler la motivation alimentaire des animaux en fonction de l'état des végétations à faire consommer lors de chaque étape du cycle annuel. Il repose dans une large mesure sur l'expérience acquise au contact du terrain et aussi sur l'habileté dans l'usage de la contrainte (clôtures). Avec la bonne maîtrise de l'agnelage en plein air, l'accent est ainsi mis sur la technicité requise pour la conduite d'un tel élevage, ce qui rejoint les observations de Benoit et al (1997) sur les élevages extensifs du Montmorillonnais.

Le recours à un animal prolifique, associé à la bonne maîtrise de son élevage, devrait garantir les marges économiques reconnues indispensables dans les élevages ovins utilisateurs de parcours (Thériez et al 1996, Benoit et al 1997, Benoit et al 1999). Il reste à affiner les conditions à remplir pour un élevage des jeunes le plus autonome possible et pour la production de carcasses conformes à la demande du marché dans le cadre d'une phase de finition la plus brève possible.

Les éléments résumés ci-dessus constituent un ensemble de connaissances et d'outils utiles au diagnostic, à la prévision et à la décision pour l'élevage sur les territoires difficiles (Béranger et al 1998). Ils peuvent prétendre au transfert dans de nombreux milieux pastoraux comparables à condition que les acteurs de terrain aient le niveau de compétence requis pour maîtriser les subtils équilibres mis en œuvre dans un tel élevage à finalités multiples. Elaborés avec le souci de la réduction des charges tout en maintenant un bon niveau de productivité animale (Thériez et al 1997), les résultats actuels du programme conduit au domaine de La Fage peuvent être associés aux repères concernant la recherche de pistes de développement d'un élevage moderne, viable, vivable et respectueux des dynamiques écologiques sur parcours. Ils peuvent alimenter une réflexion sur le rôle que pourrait jouer une plus grande valorisation des parcours, friches ou montagnes dans de nombreuses régions sèches et/ou chaudes, indépendamment du pays concerné. Concernant l'élevage français, ils devraient contribuer à l'amélioration de sa compétitivité face à la concurrence internationale, européenne et surtout océanienne (Garrigues 2004).

\section{Remerciements}

Les auteurs tiennent à remercier les personnes qui se sont impliquées dans ce programme, pour le domaine de la Fage Jean-Luc Escach et Claude Racine en particulier, et pour l'UMR ERRC de Montpellier Robin Dumas, Sébastien Douls et Michèle Inesta.

\section{Références}

Amalbert M., Orange A., 1924. Le Mérinos d'Arles. F. Genre et Cie (Ed), Antibes, France, $85 \mathrm{p}$.

Baumont R., Prache S., Meuret M., MorandFehr P., 2000. How forage characteristics influence behaviour and intake in small ruminants: a review. Cahier Options Méditerranéennes, 5, 11-25.

Benoit M., Laignel G., Liénard G., 1999. Facteurs techniques, cohérence de fonctionnement et rentabilité en élevage ovin allaitant. Exemples du Massif Central Nord et du Montmorillonnais. Renc. Rech. Rum., 6, 19-22.

Benoit M., Laignel G., Liénard G., Dedieu B., Chabosseau J.M., 1997. Eléments de réussite économique des élevages ovins extensifs du Montmorillonnais. INRA Prod. Anim., 10 (5), 349-362.

Béranger C., Gibon A., Flamant JC., 1998. Développement durable en élevage et développement territorial. Renc. Rech. Rum., 5, 155-160.

Biagini F., Molénat G., Hubert D., 2003. Production ovine en zone difficile : une recherche à l'INRA sur la finition à l'herbe des agneaux nés sur parcours. Rapport de stage, ESA Purpan, Novembre 2003, 45p. + annexes.

Boissy A., Bouix J., Orgeur P., Poindron P., Bibé B., Le Neindre P., 2005. Genetic analysis of emotional reactivity in sheep: effects of the genotypes of the lambs and of their dams. Genet. Sel. Evol., 37, 381-401.

Boissy A., Nowak R., Orgeur P., Veissier I., 2001. Les liens sociaux chez les ruminants d'élevage : limites et moyens d'action pour favoriser l'intégration de l'animal dans son milieu. INRA Prod. Anim., 14 (2), 79-90.
Bouix J., 1992. Adaptation des ovins aux conditions de milieu difficiles. In : Eléments de génétique quantitative et application aux productions animales. INRA Prod. Anim., hors série, 179-184.

Bouix J., Jacquin M., Foulquié D., Autran P., Guillouet P., Hubert D., Bibé B., 2002. Genetic effects on sheep production in harsh environment and extensive management system. Proc. $7^{\text {th }}$ World Congress G.A.L.P., Montpellier, France, 441-444.

Bouix J., Prud'hon M., Molénat G., Bibé B., Flamant J.C., Maquère M., Jacquin M., 1985. Potentiel de prolificité des brebis et systèmes de production utilisateurs de parcours. Résultats expérimentaux. $10^{\mathrm{e}}$ Journ. Rech. Ovine et Caprine, INRA-ITOVIC-SPEOC, Paris, France, 252-291.

Chenost M., Molénat G., 1996. Perspectives d'utilisation des fourrages grossiers dans les zones méditerranéennes et tropicales. Ann. Zootech., 45, Suppl., 137-139.

Garrigues B., 2004. 2003 : l'année économique ovine. Le dossier Economie de l'élevage, Institut de l'Elevage (Ed), 334, 57p. + annexes.

Gibon A., 1981. Pratiques d'éleveurs et résultats d'élevage dans les Pyrénées centrales. Thèse de Sciences et Techniques INA-PG, Septembre $1981,117 \mathrm{p}$. + annexes.

Griffon L., Jullien E., Lemaire V., Tiphine L., 2005. Bilan du contrôle de performances ovins allaitants - campagne 2004. Institut de l'Elevage (Ed), collection résultats, $101 \mathrm{p}$.

Groupe de recherches INRA-CNRS, 1979. Les parcours des Grands Causses : contraintes et possibilités techniques d'une mise en valeur par l'élevage ovin. In : Molénat G., Jarrige R.
(Eds), Utilisation par les ruminants des pâturages d'altitude et parcours méditerranéens, INRA Editions, Paris, France, 257-334.

Hubert D, Grandjanny M., 1989. Pour une gestion écologique des pelouses pâturées caussenardes : premiers résultats d'une expérience. Ann. Parc National des Cévennes, 4, 191-227.

Jacquin M., Allain D., Bouix J., Foulquié D., Autran P, Bibé B., 2002. Lamb survival, coat surface temperature and growing performances in relation to birthcoat type among different genotype breeds raised under permanent exposure outdoors. Proc. $7^{\text {th }}$ World Congress G.A.L.P. Montpellier, France, 379-382.

Jarrige R., 1979. Utilisation des pâturages des milieux défavorisés : Essai de conclusions. In : Molénat G., Jarrige R. (Eds), Utilisation par les ruminants des pâturages d'altitude et parcours méditerranéens. INRA Editions, Paris, France, 541-565.

Lambert B., Senn O., Molénat G., Thiault M., 1985. Le Buech, un exemple de recherche développement pour l'élevage ovin dans les Préalpes sèches. $10^{\mathrm{e}}$ Journ. Rech. Ovine et Caprine, INRA-ITOVIC-SPEOC, 445-459.

Langlet A., 1979. Recherches sur l'adaptation des systèmes agricoles à la sécheresse, exemple des Causses. In : INRA, L'eau et la production agricole. SEI INRA (Ed), Versailles, France.

Langlet A., Hubert D., 1985. Végétations et ressources dans les zones sèches difficiles : caractérisation et possibilités d'utilisation. $10^{\mathrm{e}}$ Journ. Rech. Ovine et Caprine, INRA-ITOVICSPEOC, 57-82.

Leclerc B., Lecrivain E., Béchet G., 1978. Régime alimentaire d'un troupeau de brebis conduit en extensif sur les parcours du Larzac. 
$4^{\mathrm{e}}$ Journ. Rech. Ovine et Caprine, INRAITOVIC-SPEOC, 155-165.

Lecomte L., Caraguel B., François D., Gibon A., 1996. Entretenir l'espace avec un élevage ovin prolifique : création d'un groupement pastoral adoptant des transhumances en Isère et Var. Renc. Rech. Rum., 3, 55-58.

Louault F., de Montard F. X., Brelurut A., Thériez M., Pailleux J. Y., Benoit M., Liénard G., 1998. Extensification en élevage ovin par agrandissement des surfaces. Adaptation de la gestion des prairies. Fourrages, 154, 217-237.

Molénat G., Béchet G., Hubert D., Maquère M., 1975. Value of a range grazing for sheep on a calcareous plateau in France. Proc. $6^{\text {th }}$ Gen. Meet. Europ. Grassland Fed., Madrid, Espagne, 163-167.

Molénat G., Chenost M., 1996. Introduction à l'utilisation des ressources fourragères par les ruminants en régions chaudes. Ann. Zootech., 45, Suppl., 9-10.

Molénat G., Dureau R., Fabre P., Lambertin M., 2003. Les «herbes» des troupeaux ovins transhumants de Crau. Multiples dimensions d'une gestion pastorale et fourragère. Fourrages, $176,437-461$.

Molénat G., Flamant J-C., Thiault M., Hubert D., 1976. Utilisation des parcours de la France méridionale. Fourrages, 67, 79-103.
Molénat G., Hubert D., Poissonet J., 1999. Raisonner les innovations techniques pour les systèmes extensifs des régions chaudes. In : Dynamics and sustainability of mediterranean pastoral systems. Cahiers Options Méditerranéennes, 39, 157-160.

O’Reagain P. J., Schwartz J., 1995. Dietary selection and foraging strategies of animals on rangeland. Coping with spatial and temporal variability. In : Journet M., Grenet E., Farce M. H., Thériez M, Demarquilly C. (Eds). Recent Developments in the nutrition of Herbivores. Proc. IVth Int. Symp. Nut. Herbivores, INRA Editions, Paris, France, 407-423.

Prache S., 1987. Possibilité de réduction des coûts de production des agneaux d'herbe. Forum Fourrages, Auvergne 1986. D. Micol (Ed), 289299.

Roggero P.P., Bellon S., Rosales M., 1996. Sustainable feeding systems based on the use of local resources. Ann. Zootech., 45, Suppl., 105-118.

Rouquette J. L., Caillette, J., Cazalot G., Léger F., Servière G., Tchakérian E., 1997. Quelques domaines clés du projet Ovins 2001. Propositions d'éleveurs «porteurs d'avenir». Renc. Rech. Rum., 4, 19-22.

Talamucci P., Pardini A., 1999. Pastoral systems dominated by fodder crops, harvesting and grazing. Cahiers Options Méditerranéennes, 39, $29-44$
Tchamitchian L., Lefevre C., Brunel J.C., Bibé B., Ricordeau G. 1986. Development of a new synthetic prolific line of sheep (INRA 401). Proc. $3^{\text {rd }}$ World Congress G.A.L.P., Madrid, Espagne, IX, 535-540.

Thériez M., Brelurut A., Pailleux J. Y., Benoit M., Liénard G., Louault F., de Montard F. X. 1997. Extensification en élevage ovin viande par agrandissement des surfaces fourragères. Résultats zootechniques et économiques de cinq ans d'expérience dans le Massif Central Nord. INRA Prod. Anim., 10 (2), 141-152.

Thériez M., Molénat G., Landais E., 1996. Feeding and management of prolific sheep. Under extensive management : the Romanov experiment in France. In: Prolific sheep, M.H Fahmy Ed., CAB International (Ed), University Press, Cambridge, UK, 429-451.

Thériez M., Petit M., Martin-Rosset W., 1994 Caractéristiques de la conduite des troupeaux allaitants en zones difficiles. Ann. Zootech., 43, 33-47.

Thorhallsdottir A. G., Provenza F. D., Balph D. F., 1990. Ability of lambs to learn about novel foods while observing or participating with social models. Appl. Anim. Behav. Sci., 25, 25-35.

\section{Résumé}

Sur le Causse du Larzac, plusieurs Unités de recherche de l'INRA collaborent à la conception d'un élevage ovin allaitant performant sur pelouses sèches dans le but de concilier les fortes exigences zootechniques avec l'entretien d'un milieu ouvert. La végétation pastorale est au cour du système de production expérimental ; son renouvellement doit être assuré et son usage privilégié comme ressource alimentaire principale d'un élevage qui doit tendre vers la durabilité. Les travaux ont été menés selon plusieurs orientations complémentaires : études génétiques autour de l'animal productif adapté ; élaboration de pratiques d'élevage en plein air ; raisonnement des calendriers et des conduites de pâturage valorisant la pelouse avec des adaptations innovantes.

C'est ainsi qu'au domaine de La Fage, des brebis prolifiques INRA 401, agnelant en début de printemps, sont élevées en plein air intégral sur parcours et alimentées majoritairement par la végétation spontanée dont une faible proportion a été fertilisée. Différentes options d'exploitation des ressources ont été étudiées. Pour s'affranchir pendant la lactation d'apports d'aliments complémentaires exogènes, les brebis disposent à partir de la mi-avril, des surfaces fertilisées qui, avec 3 à $\mathbf{4}$ semaines d'avance sur les pelouses natives, offrent une herbe abondante de qualité, à base de graminées. Ces surfaces, pâturées en rotation (4 parcelles) pendant une trentaine de jours, jusque vers le $20 \mathrm{mai}$, selon deux cycles successifs à raison de $17 \mathrm{brebis} / \mathrm{ha}$, ont permis des gains de poids journaliers moyens de $268 \mathrm{~g}$ à $295 \mathrm{~g}$ selon les années pour des agneaux dont les $2 / 3$ étaient allaités doubles. Les croissances ont ensuite pu être maintenues aux alentours de $250 \mathrm{~g} / \mathrm{j}$ jusqu'au sevrage, à la fin juin, en passant sur les parcours natifs, alors pratiquement au stade optimum. Pour ce faire sur cette période, le chargement était de 2 à 3 brebis/ha, la fréquence de changement de parcelle de 6 à 8 jours et l'herbe était nouvelle à chaque changement.

Sur une série de 14 ans, la production printanière des pelouses natives a été en moyenne de 1 t MS/ha et celle des pelouses fertilisées de 4,4 t. Dans ces conditions, il a suffi de $6 \%$ de surface fertilisée pour obtenir les performances ci-dessus.

De la mi-avril jusqu'en décembre-janvier les brebis s'alimentent exclusivement sur les parcours, ce qui représente $68 \%$ de leurs besoins annuels en énergie calculés. Le reste correspond à des aliments distribués en hiver, du foin produit sur le domaine (18\%) et de l'aliment concentré pendant la gestation $(14 \%)$. Outre la perspective d'une forte autonomie fourragère, ce programme a montré la possibilité de maintenir le milieu ouvert, de maîtriser les espèces végétales envahissantes et de préserver la biodiversité végétale au moyen d'une exploitation des parcours en rotation dans des parcs avec des chargements instantanés élevés.

La race INRA 401, en phase d'absorption d'un troupeau de brebis $1 / 2$ sang Romanov, a révélé dans ce milieu de bonnes aptitudes de production et d'adaptation tout comme elle a été propice à l'évaluation des modalités de gestion des pâturages.

Dans l'état actuel du programme, les résultats obtenus et les solutions techniques dégagées, dans les domaines de la génétique ovine, de la conduite des troupeaux et de l'exploitation des pâturages, doivent pouvoir éclairer aussi bien le choix et la sélection des races que les démarches prospectives de mise en valeur durable des zones pastorales difficiles ou encore le corpus des conseils techniques aux éleveurs des régions concernées. 


\begin{abstract}
For a productive and sustainable sheep breeding on rangeland: an experimental system on the Causse du Larzac

Efficient animal production together with environmental care, are both the global goals of the sheep production system reported here. The challenge was to succeed in establishing a perennial system of lamb production with prolific ewes fed mainly from dry steppic rangeland resources from a calcareous area of the south part of France.
\end{abstract}

A long term experiment was settled at the INRA Domaine de La Fage on the Causse du Larzac $\left(44^{\circ} \mathrm{N}-3^{\circ} \mathrm{E} ; 800 \mathrm{~m}\right.$ a.s.l.) in order to find a suitable animal breed and to elaborate management rules both for the sheep living outside and for innovative pastoral use of a spontaneous pasture. A multidisciplinary programme was developed according to the following objectives : i) reach the highest feeding autonomy from the rangeland grass, ii) succeed in the year to year renewal of the pasture, iii) achieve good growth rates for twin suckled lambs, iv) protect the countryside and maintain plant diversity, and v) limit inputs and costs.

In this context, suckling ewes of the INRA 401 breed are reared outside all year round. They lamb in the early spring and are fed mainly from rangeland pasture. In order to reduce supplements bought outside, the grass productivity of a limited area, i.e. $6 \%$ of the rangeland was increased by means of fertilisers $\left(65 \mathrm{~kg} \mathrm{~N} / \mathrm{ha} / \mathrm{year}+120 \mathrm{~kg} \mathrm{P}_{2} \mathrm{O}_{5} /\right.$ ha every 3 years). Measured over 14 successive years, the average pasture yields were $1.0 \mathrm{t} \mathrm{DM/ha}$ on native rangeland and $4.4 \mathrm{t} / \mathrm{ha}$ on the fertilised one.

A comparison of four ways of combined use of native and fertilised grass in the spring was performed with ewes suckling an average of 1.5 lambs/dam. Average lambs'growth were 268 to $295 \mathrm{~g} /$ day on fertilised paddocks rotationally grazed (2 grazing cycles on 4 paddocks) from mid April until May 20 ${ }^{\text {th }}$, with the stocking rate being 15-20 ewes/ha. Growth rates until weaning (end of June) were then kept around $250 \mathrm{~g}$ daily when the suckling ewes grazed native rangeland areas all preserved from previous grazing (6 paddocks, stocking rate 2-3 ewes/ha). Electric fences allowed rapid rotational grazing, the ewes staying no more than 4 days at the same place on fertilised grass and 8 days on native grass. From summer rise until January, the flock grazed mature grass as well as refusals from previous grazings. The grazing procedures ensured environmental care and control of invading vegetal species as a result of permanent rotational grazing and high instant stocking rates on mature vegetations. In this experiment, the rangeland resource was the only food offered to the ewes from mid-April until December-January and it represented $68 \%$ of their total yearly energy intake. The remaining $32 \%$ was made of hay from the farm $(18 \%)$ and concentrate $(14 \%)$ given at the end of pregnancy.

The INRA 401 sheep showed productive and adaptative abilities. It can be considered a suitable breed for the sustainable valorisation for harsh and less favoured areas as well as a good tool to test different ways of pasture management.

MOLENAT G., FOUlQUIE D., AUTRAN P., BOUIX J., HUBERT D., JACQUIN M., BOCQUIER F., BIBBE B., 2005. Pour un élevage ovin allaitant performant et durable sur parcours : un système expérimental sur le Causse du Larzac. INRA Prod. Anim., 18, 323-338. 